\title{
Topology of covers and the spectral theory of geometric operators
}

\author{
Steven Hurder
}

\section{Introduction}

For a compact smooth manifold $M$, the spectrum $\sigma\left(\mathcal{D}_{M}\right) \subset \mathbf{R}$ of a symmetric elliptic differential operator $\mathcal{D}_{M}$ acting on the sections of an Hermitian vector bundle $\mathbf{E}_{M} \rightarrow M$ is discrete, and the points $\lambda \in \sigma\left(\mathcal{D}_{M}\right)$ correspond to smooth "eigensections" of $\mathbf{E}_{M}$ of finite multiplicity. If the manifold $M$ is complete but non-compact, the action of $\mathcal{D}_{M}$ on the compactly supported sections of $\mathbf{E}_{M}$ is still essentially self-adjoint, and the spectrum can be defined as the points $\lambda \in \mathbf{R}$ so that $\mathcal{D}_{M}-\lambda$ does not admit a densely-defined left or right (bounded) inverse. In this situation, the spectrum of $\mathcal{D}_{M}$ can a priori be any closed subset of R. A point $\lambda \in \sigma\left(\mathcal{D}_{M}\right)$ which is isolated must correspond to an eigensection in $\mathcal{H}\left(\mathbf{E}_{M}\right) \cap C^{\infty}\left(\mathbf{E}_{M}\right)$. In general, though, a cluster point $\lambda \in \sigma\left(\mathcal{D}_{M}\right)$ need not coincide with an eigensection, but rather to a sequence of "approximate eigensections" which eventually vanish on compact sets. We thus expect there to be a correspondence between the essential spectrum $\sigma_{e}\left(\mathcal{D}_{M}\right)$, especially the topology of its derived set, and the behavior of $M$ "near infinity".

A typical example of how the essential spectrum of elliptic operators on open manifolds can be related to geometric properties was discovered by R. Brooks $[8,9]$. Brooks considered a normal covering $\tilde{M}_{\Gamma}$ of a compact manifold $M$ with Galois covering group $\Gamma$, and showed that the essential spectrum $\sigma_{e}\left(\Delta_{\Gamma}\right)$ of the Laplacian on functions on $\tilde{M}_{\Gamma}$ contains 0 if and only if the group $\Gamma$ is amenable. Equivalently, $\Gamma$ is non-amenable if and only if there is a gap about the origin in the spectrum of the Laplacian. Brooks' methods are based on the relation between the Cheeger isoperimetric constant for $\tilde{M}_{\Gamma}$ and the spectrum of the Laplacian.

Coarse cohomology theory, introduced by John Roe [47, 48, 49], gives a precise meaning to the concept of the "topology at infinity" for an open complete manifold. Roe showed that if there is a gap containing zero for the spectrum of a uniform geometric operator $\mathcal{D}$ on a complete, non-compact even-dimensional

\footnotetext{
AMS classification: Primary 58G25, 57R30, 58F18, 35P15

Supported in part by NSF Grants DMS 89-02960 and DMS 91-03297

This paper is in final form and no version of it will be submitted for publication elsewhere.
} 
Riemannian manifold of bounded geometry, then the "exotic analytic index" of the operator must vanish (Proposition 4.33 [49].) For odd dimensional manifolds, the conclusion is stronger, that if there is a gap anywhere in the spectrum of the operator, then its exotic analytic index class vanishes (Proposition 4.46, [49].) Roe established topological obstructions to the existence of a gap in the spectrum of geometric operators (Proposition 5.21, [49]) by combining these vanishing results with an "exotic index theorem" which relates the exotic analytic index with the coarse cohomology of the ambient manifold.

In this paper we further investigate the dependence of the spectrum of an operator $\tilde{D}$ on the topology at infinity of an open manifold of odd dimension, either in the case of a covering $\tilde{M}_{\Gamma}$ of a compact manifold $M$, or for a leaf $L$ of a foliation of a compact manifold $V$. The leafwise operator $\tilde{D}$ will be an essentially self-adjoint, elliptic, first-order pseudo-differential operator which is a relatively compact perturbation of the lift $\mathcal{D}_{\Gamma}$ of a first order geometric (or generalized Dirac) operator $\mathcal{D}_{M}$ on $M$, as in Definition 3.2. Our approach combines the "Vafa-Witten method" (section III, [52] \& section 3, [2]) with the foliation index theorem for leafwise Toeplitz operators $([13,23,24,25])$ to obtain topological obstructions to the existence of a gap in the spectrum $\sigma(\tilde{\mathcal{D}})$. The Vafa-Witten method is compared with the coarse cohomology method for detecting gaps in Remark 7.8 and Example 3 in section 7.

Perhaps the most important aspect of our method is that it also provides estimates on the spectral density function for the operator $\tilde{\mathcal{D}}$ in terms of the topology at infinity of $\tilde{M}_{\Gamma}$ and of the symbol of the operator $\mathcal{D}_{M}$ on $M$. The spectral density estimates introduce a new concept for a finitely-generated group $\Gamma$, Definition 2.8, the non-commutative isoperimetric function $\mathcal{I}_{\Gamma, u}(\epsilon)$. The function $\mathcal{I}_{\Gamma, u}(\epsilon)$ depends upon the choice of an element of odd K-theory $u \in$ $K^{1}(B \Gamma)$, and measures how "efficiently" the K-theory class $u$ can be realized on the open manifold $\tilde{M}_{\Gamma}$ in terms of volume. For $\epsilon>0$ sufficiently large and $u \neq 0$, $\mathcal{I}_{\Gamma, u}(\epsilon)>0$. If $B \Gamma$ is realized by a finite $\mathrm{CW}$ complex, then the equivalence class of the function $\epsilon \mapsto \mathcal{I}_{\Gamma, u}(\epsilon)$ is a group invariant of $\Gamma$ (Proposition 2.9.) The terminology for $\mathcal{I}_{\Gamma, u}(\epsilon)$ is chosen due to an analogy with the function $\mathcal{I}_{\Gamma}(\epsilon)$ which gives the reciprocal of the minimum volume of a region in $\tilde{M}_{\Gamma}$ having isoperimetric constant $\epsilon$.

Here is our main result for the case of coverings:

THEOREM 1.1 • Let $M$ be a compact orientable odd-dimensional Riemannian manifold with fundamental group $\Lambda=\pi_{1}\left(M, y_{0}\right)$.

- For a quotient group $\rho: \Lambda \rightarrow \Gamma$, let $\pi: \tilde{M}_{\Gamma} \rightarrow M$ be the associated normal covering.

- Fix an element of odd K-theory $u \in K^{1}(B \Gamma)$.

- Given a first-order, symmetric, geometric operator $\mathcal{D}_{M}$ acting on the sections of a Hermitian vector bundle $\mathbf{E}_{M} \rightarrow M$, let $\mathcal{D}_{\Gamma}: C_{c}^{1}\left(\mathbf{E}_{\Gamma}\right) \rightarrow C_{c}^{1}\left(\mathbf{E}_{\Gamma}\right)$ denote the lifted operator acting on the compactly supported sections of the lifted Hermitian bundle $\mathbf{E}_{\Gamma}=\pi^{!}\left(\mathbf{E}_{M}\right) \rightarrow \tilde{M}_{\Gamma}$. 
- $\tilde{\mathcal{D}}$ is a $\Gamma$-invariant, relatively compact perturbation of $\mathcal{D}_{\Gamma}$ as in Definition 3.2.

Then there exists a constant $\kappa(\tilde{\mathcal{D}})>0$, defined in $(9)$, which depends on the Riemannian geometry of $M$ and the perturbation $\tilde{\mathcal{D}}$, so that for all $\lambda \in \mathbf{R}$ and all $\epsilon>0$,

$$
\operatorname{Tr}_{\Gamma}\left(\left\{\chi_{[\lambda, \lambda+\epsilon)}(\tilde{\mathcal{D}})\right\}\right) \geq \frac{1}{4} \cdot\left|\left\langle c h^{*}\left(B \rho^{*} u\right), c h_{*}\left[\mathcal{D}_{M}\right]\right\rangle\right| \cdot \mathcal{I}_{\Gamma, u}(\epsilon / 4 \kappa(\tilde{\mathcal{D}}))
$$

where

- $\operatorname{Tr}_{\Gamma}$ is the $\Gamma$-trace of Atiyah [1],

- $\chi_{[\lambda, \lambda+\epsilon]}(\tilde{\mathcal{D}})$ is the spectral projection associated to the characteristic function $\chi[\lambda, \lambda+\epsilon]$

- the pairing in (1) is the (integral) odd Toeplitz index of the compression of the unitary multiplier for $B \rho^{*} u$ with the positive projection of $\mathcal{D}_{M}$.

In particular, if $\left\langle\operatorname{ch}^{*}\left(B \rho^{*} u, c h_{*}\left[\mathcal{D}_{M}\right]\right\rangle \neq 0\right.$ for some $u \in K^{1}(M)$, and $\mathcal{I}_{\Gamma, u}(\epsilon)>0$ when $\epsilon>0$, then the spectrum $\sigma(\tilde{\mathcal{D}})=\mathbf{R}$.

The number $\operatorname{Tr}_{\Gamma}\left(\left\{\chi_{[\lambda, \lambda+\epsilon)}(\tilde{\mathcal{D}})\right\}\right)$ is the "average spectral density" for the operator $\tilde{\mathcal{D}}$ in the interval $[\lambda, \lambda+\epsilon)$. If the spectrum of $\tilde{\mathcal{D}}$ is isolated in this interval, then $\operatorname{Tr}_{\Gamma}\left(\left\{\chi_{[\lambda, \lambda+\epsilon)}(\tilde{\mathcal{D}})\right\}\right)$ is the integral over a fundamental domain in $\tilde{M}_{\Gamma}$ of the $\Gamma$-periodic function $\sum_{n}\left\|f_{n}\right\|^{2}$, where $\left\{f_{n}\right\}$ is an orthogonal basis for the eigensections of $\tilde{\mathcal{D}}$ in $[\lambda, \lambda+\epsilon)$. The result is a type of dimension: for a compact manifold, this integral will be the dimension of the sum of the eigenspaces in this interval. More generally, it is an average density of the eigenspaces in the interval $[\lambda, \lambda+\epsilon)$, which makes sense whether the spectrum is isolated or not. (For more discussion of the interpretation of this number, see $[1,5,21,37]$.)

A fundamental point of Theorem 1.1 is that the function class of the righthand-side of (1) is a topological invariant of the symbol of the operator $\mathcal{D}_{M}$ and the K-theory class $u$, so that when the index pairing is non-trivial we obtain a topologically determined lower bound on the $\Gamma$-spectral density function for the $\Gamma$-periodic lift $\mathcal{D}_{\Gamma}$. For example, when $\Gamma \cong \mathbf{Z}^{n}$ for $n$ odd and $u$ is the top odd dimensional K-theory generator, then $\mathcal{I}_{\Gamma, u}(\epsilon) \sim \epsilon^{n}$ for $\epsilon$ small.

Results which link topology to spectral properties of operators ultimately rely on an appropriate index theorem, formulated for the context under consideration. In the present paper, the relevant index theorem is that for Toeplitz operators formed from the spectral projections associated to self-adjoint operators on normal coverings as in [34]. This "odd" index theorem is based on Connes' index theorem for measured foliations [13] and the foliation spectral flow theory for measured foliations developed by Douglas, Hurder and Kaminker $[23,24,25]$. 
The new "topological" idea of the present work is based on the fact that the index theory of self-adjoint operators requires the choice of a Toeplitz multiplier function, and the index of the resulting Fredholm operator depends upon the geometric properties of this multiplier. Considering the effect of the choice of this multiplier on the index leads to the $\Gamma$-isoperimetric function in section 2 , which is a new tool for exploring the topology-at-infinity coverings and leaves of foliations. This topological data is used to detect gaps in the spectrum of an operator and to estimate its spectral density properties, by an open manifold version of the "Vafa-Witten method" relating commutator estimates to spectral flow (cf. section III, [52]; section 3, [2]; Theorem 6.4, [32]).

A preliminary form of Theorem 1.1 was announced in [35]. The formulation of the isoperimetric theory was introduced in [36]. Related results are contained in the papers $[38,39]$.

The paper [10] by U. Bunke discusses ideas which are very similar to those of this work, but in the context of a single open manifold and for geometric operators with a gap in their spectrum.

The author is indebted to Nigel Higson for explaining the ideas of "asymptotic K-theory" and discussions related to this paper, to John Roe for ongoing discussions about coarse geometry, and to Ulrich Bunke for pointing out a mistake in the proof of Theorem 4.3 in a preliminary version of this paper.

\section{Non-commutative isoperimetric functions}

The notion of almost flat vector bundles was introduced by Connes, Gromov and Moscovici for the study of the Novikov conjecture for compact manifolds $[17,18]$, motivated by the work of Gromov and Lawson [29]. These special bundles generate a subgroup $K_{a f}^{0}(M) \subset K^{0}(M)$ of the Grothendieck group of the manifold $M$, and a fundamental point is that the index of the Dirac operator paired with an element of $K_{a f}^{0}(M)$ is a topological invariant [33].

The construction of an index class for a self-adjoint elliptic operator requires pairing a spectral projection obtained from the given operator with a unitary multiplier, to obtain a generalized Toeplitz operator $[4,25]$. The work of this paper is based on estimating the index of the generalized Toeplitz operator constructed from a $\Gamma$-almost flat unitary map for $M$, where $\Gamma$ is an infinite quotient of the fundamental group of $M$. This leads to the concept of the $\Gamma$ almost flat odd K-theory, $K_{\Gamma a f}(M)$, which is the K-theory associated to the $\Gamma$-almost flat unitaries. This is just a special case of $\mathcal{F}$-almost flat odd $\mathrm{K}$ theory for a foliated manifold $(V, \mathcal{F})$. In fact, the definition of $K_{\Gamma a f}(M)$ requires formulating these ideas in the generality of foliated spaces, so there is where we begin.

Let $(V, \mathcal{F})$ be a compact foliated measure space with leaves of dimension $m$ (cf. section 2, [40]). In particular, this implies there is a finite covering of $V$ by "flow boxes" $\left\{\phi_{\alpha}: U_{\alpha} \cong \mathbf{D}^{m} \times X_{\alpha} \mid \alpha \in \mathcal{I}\right\}$ so that the intersections of the leaves of $\mathcal{F}$ with each $U_{\alpha}$ are given by the level sets (plaques) $P_{\alpha}(x)=\phi_{\alpha}^{-1}\left(\mathbf{D}^{m} \times\{x\}\right)$. Here $\mathbf{D}^{m}=(-1,1)^{m}$ and $X_{\alpha}$ is a Borel measure space. We assume there is 
given a transverse measure $\mu$ for $\mathcal{F}$, whose measure class is invariant under the transverse holonomy transformations. That is, the measure structures on the local transversals $T_{\alpha}=\phi_{\alpha}^{-1}\left(\{0\} \times X_{\alpha}\right)$ are determined by the restrictions $\mu_{\alpha}$ of $\mu$, and the local holonomy maps $\gamma_{\alpha \beta}$ from a subset of $T_{\alpha}$ to a subset of $T_{\beta}$ pulls the measure class of $\mu_{\beta}$ back to that of $\mu_{\alpha}$. For each leaf $L \subset V$, we also assume there is given a Riemannian metric $\langle\cdot, \cdot\rangle_{L}$ of bounded geometry, so that the leafwise metrics vary bounded measurable with the transverse parameter. Let $\nabla^{L}$ denote the associated Riemannian connection on the leaf $L$, and let $\nabla^{\mathcal{F}}$ denote the collection of all the leafwise connections.

A Hermitian vector bundle $\mathbf{E} \rightarrow V$ is a foliated Hermitian flat bundle if for each foliation chart $U_{\alpha}$, there is a trivialization $\Phi_{\alpha}: \mathbf{E} \mid U_{\alpha} \cong \mathbf{C}^{N} \times \mathbf{D}^{m} \times X_{\alpha}$, such that

- On the overlap of $U_{\alpha} \cap U_{\beta}$, the transition function

$$
\Phi_{\beta}^{-1} \circ \Phi_{\alpha}: \mathbf{C}^{N} \times \phi_{\alpha}\left(U_{\alpha} \cap U_{\beta}\right) \longrightarrow \mathbf{C}^{N} \times \Phi_{\beta}\left(U_{\alpha} \cap U_{\beta}\right)
$$

is a constant Hermitian isomorphism when restricted to the "horizontal sets" $\mathbf{D}^{m} \times\{x\}$

- $\Phi_{\beta}^{-1} \circ \Phi_{\alpha}(\vec{v}, x)$ depends measurably on the parameter $x$ for all $\vec{v} \in \mathbf{C}^{N}$;

Let $\nabla^{\mathbf{E}_{L}}$ denote the leafwise Hermitian connection for $\mathbf{E}_{L}$

$U(\mathbf{E})$ denotes the $U(N)$-principal bundle of unitary fiberwise automorphisms of $\mathbf{E}$. Let $C_{\mathcal{F}}^{1}(U(\mathbf{E}))$ denote the measurable sections whose restrictions to leaves are $C^{1}$. In the case where $V=M$ with the one leaf foliation, with $L=M$, we write $C^{1}(U(\mathbf{E}))=C_{\mathcal{F}}^{1}(U(\mathbf{E}))$.

Define a $C^{1}$-pseudo-norm for $g \in C_{\mathcal{F}}^{1}(U(\mathbf{E}))$ : Let $\left\{\tilde{h}_{1}, \ldots, \tilde{h}_{N}\right\}$ be a local

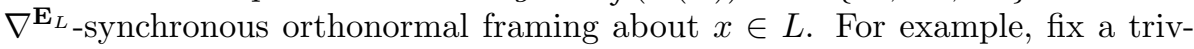
ialization $\Phi_{\alpha}: \mathbf{E} \mid U_{\alpha} \cong \mathbf{C}^{N} \times \mathbf{D}^{m} \times X_{\alpha}$ with $x \in U_{\alpha}$. Choose an Hermitian framing $\left\{\vec{v}_{1}, \ldots, \vec{v}_{N}\right\}$ for $\mathbf{C}^{N}$ for the induced metric on $\mathbf{C}^{N}$, then set

$$
\tilde{h}_{\ell}(v)=\Phi_{\alpha}^{-1}\left(\vec{v}_{\ell}, \varphi_{\alpha}(v)\right)
$$

The restriction of $\left\{\tilde{h}_{1}, \ldots, \tilde{h}_{N}\right\}$ to the plaque of $L$ containing $x$ gives a local synchronous framing.

For $g \in C_{\mathcal{F}}^{1}(U(\mathbf{E}))$, let $g_{L}$ denote the restriction to a leaf $L$. We use a synchronous framing on $L$ about $x \in L$ to express $g_{L}$ in matrix form:

$$
g_{L} \cdot \tilde{h}_{j}=\sum_{1 \leq i \leq N}\left(g_{L}\right)_{i j} \cdot \tilde{h}_{i}
$$

for local $C^{1}$-functions $g_{i j}^{L}$ defined on an open neighborhood in $L$ of $x$. Then define

$$
\|g\|_{(1)}=\sup _{L \subset V} \sup _{x \in L} \sqrt{\sum_{1 \leq i, j \leq N}\left\|\left.\nabla^{L}\left(g_{L}\right)_{i j}\right|_{x}\right\|^{2}}
$$

A map $g \in C_{\mathcal{F}}^{1}(U(\mathbf{E}))$ is admissible if $\|g\|_{(1)}<\infty$. 
DEFINITION 2.1 (cf. Definition 5.1 [33]) An almost flat odd cocycle for $(V, \mathcal{F})$ consists of the data $g^{a f}=\left\{\left(g_{i}, N_{i}\right) \mid i \geq 1\right\}$ such that for each $i \geq 1$ :

- $\mathbf{E}_{i} \rightarrow V$ is a foliated Hermitian flat bundle of dimension $N_{i}$

- $g_{i} \in C_{\mathcal{F}}^{1}\left(U\left(\mathbf{E}_{i}\right)\right)$ is an admissible map with $\left\|g_{i}\right\|_{(1)} \leq 1 / i$

- The stabilized vector bundles $\mathbf{E}_{i} \oplus \mathbf{C}^{\infty}$ are all isomorphic to a common Hermitian vector bundle $\mathbf{E}_{\infty} \rightarrow V$

- there is a continuous family of admissible maps $g_{t} \in C_{\mathcal{F}}^{1}\left(U\left(\mathbf{E}_{\infty}\right)\right)$ for $i \leq t \leq i+1$ interpolating between the stabilized sections $g_{i}$ and $g_{i+1}$.

We say that two almost flat odd cycles $\left\{g^{a f}\right\}$ and $\left\{h^{a f}\right\}$ are equivalent if there exists admissible maps $H_{i}(t) \in C_{\mathcal{F}}^{1}\left(U\left(\mathbf{E}_{\infty}\right)\right)$ interpolating between $g_{i}$ and $h_{i}$ for all $i \geq 0$.

PROPOSITION 2.2 The set of equivalence classes of almost flat odd cocycles for $(V, \mathcal{F})$ forms a group, $K_{\text {af }}^{1}(V, \mathcal{F})$, called the almost flat odd K-theory of $\mathcal{F}$.

Suppose that $M$ is a compact Riemannian manifold with fundamental group $\Lambda=\pi_{1}\left(M, y_{0}\right)$, and $\tilde{M}_{\Gamma} \rightarrow M$ is the covering associated to a surjection $\rho: \Lambda \rightarrow$ $\Gamma$, with the covering group $\Gamma$ acting on the left on $\tilde{M}_{\Gamma}$. We recall the suspension construction of a foliation associated to each action of $\Gamma$ on a measure space $X$ (cf. Chapter 5 [11]). Let $X$ denote a standard, second countable Borel measure space, with $\tilde{\mu}$ a Borel probability measure on $X$. Consider a Borel action $\varphi: \Gamma \times X \rightarrow X$ which preserves $\tilde{\mu}$. The product of the deck action on $\tilde{M}_{\Gamma}$ with the $\varphi$-action on $X$ defines an action of $\Gamma$ on $\tilde{M}_{\Gamma} \times X$. Introduce the quotient measure space,

$$
V_{\varphi}=\Gamma \backslash\left(\tilde{M}_{\Gamma} \times X\right) .
$$

The product foliation on $\tilde{M}_{\Gamma} \times X$, with typical leaf $\tilde{L}=\tilde{M}_{\Gamma} \times\{x\}$ for $x \in X$, descends to a measurable foliation denoted by $\mathcal{F}_{\varphi}$ on $V_{\varphi}$. The measure $\tilde{\mu}$ descends to a holonomy-invariant transverse measure $\mu$ for $\mathcal{F}_{\varphi}$.

Let $K_{\varphi} \subset \Lambda$ denote the subgroup of elements which act trivially on $X$ under $\varphi$, and let $\Gamma_{\varphi}=\Lambda / K_{\varphi}$ denote the quotient group. The global holonomy group of $\mathcal{F}_{\varphi}$ is the isomorphic image

$$
\Gamma_{\varphi} \stackrel{\varphi}{\cong} \mathcal{H}_{\mathcal{F}_{\varphi}} \subset \operatorname{Aut}(X)
$$

The typical leaf of $\mathcal{F}_{\varphi}$ is diffeomorphic to the covering $\tilde{M}_{\Gamma_{\varphi}}$.

The projection onto the first factor map, $\tilde{M}_{\Gamma} \times X \rightarrow \tilde{M}_{\Gamma}$, descends to a map $\pi: V_{\varphi} \rightarrow M$, and $\pi$ restricted to the leaves $\mathcal{F}_{\varphi}$ is a covering map. The Riemannian metric on $T M$ lifts via $\pi^{*}$ to a leafwise metric on $T \mathcal{F}_{\varphi}$. 
There is a natural construction of Borel measure space $\left(X_{\Gamma}, \tilde{\mu}_{\Gamma}\right)$ associated to a group $\Gamma$, equipped with a measure preserving ergodic action $\varphi$ of $\Gamma$. Endow the two-point space $\mathbf{Z}_{\mathbf{2}}=\{0,1\}$ with the " $\frac{1}{2}-\frac{1}{2}$ " probability measure, and set

$$
X_{\Gamma}=\prod_{\gamma \in \Gamma}\left(\mathbf{Z}_{\mathbf{2}}\right)_{\gamma}
$$

equipped with the product topology from the factors, and the product measure $\tilde{\mu}_{\Gamma}=\prod_{\gamma \in \Gamma} \mu_{\gamma}$.

A typical element of $X_{\Gamma}$ is a string $x=\left\{a_{\gamma}\right\}=\left\{a_{\gamma} \mid a_{\gamma} \in \mathbf{Z}_{\mathbf{2}}\right.$ for $\left.\gamma \in \Gamma\right\}$. Let $\varphi: \Gamma \times X_{\Gamma} \rightarrow X_{\Gamma}$ be the "shift" action of $\Gamma$ on $X_{\Gamma}$, defined by $\varphi\left(\delta,\left\{a_{\gamma}\right\}\right)=\left\{a_{\delta \gamma}\right\}$. The shift action is continuous, transitive, measure-preserving, ergodic and free for $\tilde{\mu}_{\Gamma}$-a.e. $x \in X_{\Gamma}$.

For each quotient group $\Lambda \rightarrow \Gamma$, introduce the foliated measure space $V_{\Gamma}=$ $\Gamma \backslash\left(\tilde{M}_{\Gamma} \times X_{\Gamma}\right)$ with foliation $\mathcal{F}_{\Gamma}$, transverse invariant measure $\mu_{\Gamma}$ and $\mu_{\Gamma}$-typical leaf isometric to $\tilde{M}_{\Gamma}$.

DEFINITION 2.3 $A$-almost flat odd cocycle for $M$ consists of the data $g^{a f}=\left\{\left(g_{i}, \mathbf{E}_{i}, N_{i}\right) \mid 0 \leq i\right\}$ which satisfy:

1. $\mathbf{E}_{0} \rightarrow M$ is the product bundle with fibers of dimension $N_{0}$

2. $\mathbf{E}_{i} \rightarrow M$ is an Hermitian flat bundle of dimension $N_{i}$ associated to a holonomy homomorphism $\Lambda \stackrel{\rho}{\rightarrow} \Gamma \stackrel{\alpha}{\rightarrow} U\left(N_{i}\right)$

3. $g_{i} \in C^{1}\left(U\left(\mathbf{E}_{i}\right)\right)$ is an admissible map with $\left\|g_{i}\right\|_{(1)} \leq 1 / i$

4. Each stabilized vector bundle $\mathbf{E}_{i} \oplus \mathbf{C}^{\infty}$ is isomorphic to a fixed Hermitian vector bundle $\mathbf{E}_{\infty}$

5. For each $i \geq 0$, there is an admissible map $g_{t} \in C_{\mathcal{F}_{\Gamma}}^{1}\left(U\left(\pi ! \mathbf{E}_{\infty}\right)\right)$ for $i \leq$ $t \leq i+1$ interpolating between the stabilized sections $\pi^{*} g_{i}$ and $\pi^{*} g_{i+1}$, where $\pi: V_{\Gamma} \rightarrow M$.

$\left[g^{a f}\right] \in K^{1}(M)$ denotes the class of the map $g_{0}$ considered as a map $g_{0}: M \rightarrow$ $U\left(N_{0}\right)$.

DEFINITION 2.4 For a quotient group $\rho: \Lambda \rightarrow \Gamma$, the $\Gamma$-almost flat odd K-theory of $M$ is the subgroup $K_{\Gamma a f}^{1}(M) \subset K^{1}(M)$ of elements $\left[g^{\text {af }}\right]$, where $g^{\text {af }}$ is a $\Gamma$-almost flat odd cocycle for $M$.

DEFINITION 2.5 The almost flat odd K-theory of $M$ is the group $K_{a f}^{1}(M)=$ $K_{\Lambda a f}^{1}(M)$ associated to the fundamental group $\Lambda$ of $M$.

Almost flat odd K-theory has the following functoriality:

PROPOSITION 2.6 Let $\Lambda \stackrel{\rho}{\rightarrow} \Gamma \stackrel{q}{\rightarrow} \Gamma^{\prime}$ be a composition of submersions. Then there is a natural map

$$
q^{!}: K_{\Gamma^{\prime} a f}^{1}(M) \rightarrow K_{\Gamma a f}^{1}(M) .
$$

In particular, for all $\rho: \Lambda \rightarrow \Gamma$, there is a map $\rho^{!}: K_{\Gamma a f}^{1}(M) \rightarrow K_{a f}^{1}(M)$. 
PROPOSITION 2.7 There is a natural map

$$
\pi^{!}: K_{\Gamma a f}^{*}(M) \rightarrow K_{a f}^{*}\left(V_{\Gamma}, \mathcal{F}_{\Gamma}\right) .
$$

which is injective on rational K-theory.

Proof: The lift of a flat bundle $\pi^{!} \mathbf{E} \rightarrow V_{\Gamma}$ is again leafwise flat, so the cocycles of Definition 2.3 lift to cocycles of Definition 2.1. The equivalence relations on the two types of cocycles coincide by construction.

The real Chern character $c h^{*}: K^{*}(M) \otimes \mathbf{R} \rightarrow H^{(*)}(M ; \mathbf{R})$ is injective, so to prove the injectivity of $\pi^{!}$it suffices to show that $\pi^{*}$ is injective on real cohomology. The existence of a transverse invariant measure $\mu_{\Gamma}$ for $\mathcal{F}_{\Gamma}$ implies that there exists an "integration along the fiber map", $\pi_{*}$, from the leafwise deRham complex for $\mathcal{F}_{\Gamma}$ to the deRham complex of $M$, with $\pi_{*} \circ \pi^{*}=I d$ on cohomology. The injectivity on cohomology, hence on real K-theory, follows from this.

There is an alternate approach to "almost flat" theory, based on the asymptotic K-theory of Connes and Higson [19, 20]. They define asymptotic K-theory groups $K_{*}^{a}(\mathcal{A})$ for an arbitrary $C^{*}$-algebra $\mathcal{A}$. This leads to a definition of "almost flat K-theory" of a space $M$ as follows: let $C^{*}(\Lambda)$ be the $C^{*}$-algebra generated by the fundamental group of $M$, then there is a natural map

$$
K_{*}^{a}\left(C^{*}(\Lambda)\right) \rightarrow K^{*}\left(C^{0}(M)\right) \cong K^{*}(M)
$$

The Connes-Higson "almost flat K-theory" is the image subgroup $\tilde{K}_{a f}^{*}(M) \subset$ $K^{*}(M)$. One can show that the group $K_{a f}^{1}(M)$ defined above is the subgroup of $\tilde{K}_{a f}^{1}(M)$ generated by the $K_{a f}$-cocycles which are represented by finite-dimensional flat bundles over $M$. The estimates obtained in our work, especially (1), requires forming Toeplitz compressions with automorphisms of finite-dimensional bundles.

For a manifold $V$ with a topological foliation $\mathcal{F}$, the foliation groupoid $\mathcal{G}_{\mathcal{F}}$ plays the rôle of the fundamental group $\Lambda$ for a compact manifold $M$ (cf. Haefliger, [31]). Corresponding to the group $C^{*}$-algebra $C^{*}(\Lambda)$, there is the foliation $C^{*}$-algebra $C^{*}(V, \mathcal{F})$ introduced by Connes $[13,14]$, which is the $C^{*}$-completion of the convolution algebra of $\mathcal{G}_{\mathcal{F}}$. The Connes-Higson asymptotic K-functor can be applied applied to $C^{*}(V, \mathcal{F})$ to obtain an alternate definition of almost flat theory for foliations:

$$
\tilde{K}_{a f}^{*}(V, \mathcal{F})=K_{*}^{a}\left(C^{*}(V, \mathcal{F})\right)
$$

It is natural to inquire when the subgroup $K_{a f}^{*}(V, \mathcal{F}) \subset \tilde{K}_{a f}^{*}(V, \mathcal{F})$ is the full group, and whether the difference of these K-groups depends upon the geometry of the foliation. The answer is unknown to the author in both cases.

We next introduce the non-commutative isoperimetric function. Fix $u \in$ $K^{1}(M)$. Realize the classifying space of the discrete group $\Gamma$ with a simplicial space $B \Gamma$ endowed with a compatible Riemannian metric on the simplices (cf. $[26])$. For each $\epsilon>0$, let $D_{\Gamma, u}(\epsilon)$ denote the minimum dimension of a Hermitian 
flat bundle $\mathbf{E}_{\epsilon} \rightarrow B \Gamma$ so that $u$ is represented by a fiberwise unitary $g_{\epsilon} \in$ $C^{1}\left(U\left(\mathbf{E}_{\epsilon}\right)\right)$ with $\left\|u_{\epsilon}\right\|_{(1)} \leq \epsilon$. If no such bundle exists, set $D_{\Gamma, u}(\epsilon)=\infty$.

\section{DEFINITION 2.8 (Non-commutative $\Gamma$-isoperimetric function) For} $\epsilon>0$, set

$$
\mathcal{I}_{\Gamma, u}(\epsilon)=\frac{1}{D_{\Gamma, u}(\epsilon)}
$$

Introduce an equivalence relation on positive functions, where $f \sim g$ if there exists a constant $a>0$ such that

$$
g\left(\frac{\epsilon}{a}\right) \leq f(\epsilon) \leq g(a \epsilon) \quad \text { for all } \epsilon>0 .
$$

PROPOSITION 2.9 Let $\Gamma \cong \pi_{1}\left(B, b_{0}\right)$ where $B$ is a compact simplicial space with contractible universal covering. Then for each $u \in K^{1}(B \Gamma)$, the class of $\mathcal{I}_{\Gamma, u}(\epsilon)$ is a topological invariant.

Proof. Let $\tilde{\Gamma}$ have classifying space $\tilde{B} \simeq B \tilde{\Gamma}$ a compact simplicial complex. An isomorphism $\theta: \tilde{\Gamma} \rightarrow \Gamma$ induces a homotopy equivalence $B \theta: \tilde{B} \rightarrow B$, and we set $[\tilde{u}]=B \theta^{*} u \in K^{1}(\tilde{B})$. The assertion of the proposition is that $\mathcal{I}_{\tilde{\Gamma}, \tilde{u}} \sim \mathcal{I}_{\Gamma, u}$.

There exists finite simplicial subdivisions of $B$ and $\tilde{B}$ so that $B \theta$ is homotopic to a simplicial map. By the compactness of $\tilde{B}$ there is a constant $A>0$ so that

$$
\|f \circ B \theta\|_{(1)} \leq A \cdot\|f\|_{(1)}
$$

for all piecewise smooth maps $f: \tilde{B} \rightarrow \mathbf{R}$. Thus, if $\mathbf{E} \rightarrow B$ is a Hermitian flat bundle and $g \in C^{1}(U(\mathbf{E}))$ is an admissible map with $\|g\|_{(1)} \leq \delta$, then $B \theta^{*} g \in C^{1}\left(U\left(B \theta^{!} \mathbf{E}\right)\right)$ is an admissible map with $\|g\|_{(1)} \leq A \delta$.

Conversely, the inverse map $\theta^{-1}: \Gamma \rightarrow \tilde{\Gamma}$ determines a simplicial map $B\left(\theta^{-1}\right): B \rightarrow \tilde{B}$ for which there is a similar constant $\tilde{A}>0$.

Take $a=\max \{A, \tilde{A}\}$. Then note that if $u$ can be realized with an automorphism $g$ of a bundle of dimension $N$ and $\|g\|_{(1)} \leq \epsilon$, then via $B\left(\theta^{-1}\right)$ we obtain that $[\tilde{u}]$ can be realized with $\tilde{g}=B\left(\theta^{-1}\right)^{*} g$ having $\|\tilde{g}\|_{(1)} \leq a \epsilon$. The reciprocal relation also holds, hence

$$
D_{\Gamma, u}(\epsilon / a) \leq D_{\tilde{\Gamma}, \tilde{u}}(\epsilon) \leq D_{\Gamma, u}(a \epsilon)
$$

which implies that $\mathcal{I}_{\tilde{\Gamma}, \tilde{u}} \sim \mathcal{I}_{\Gamma, u}$.

There is a similar definition of the non-commutative isoperimetric function for foliated manifolds. Let $(V, \mathcal{F})$ be a compact foliated measure space. Fix $u \in K_{a f}^{1}(V, \mathcal{F})$ represented by a fiberwise Hermitian automorphism $g \in C_{\mathcal{F}}^{1}(\mathbf{E})$. For each $\epsilon>0$, let $D_{\mathcal{F}, u}(\epsilon)$ denote the minimum dimension of a foliated Hermitian flat bundle $\mathbf{E}_{\epsilon} \rightarrow V$ so that $u$ is represented by a fiberwise unitary $g_{\epsilon} \in C_{\mathcal{F}}^{1}\left(U\left(\mathbf{E}_{\epsilon}\right)\right)$ with $\left\|g_{\epsilon}\right\|_{(1)}<\epsilon$. If no such bundle exists, set $D_{\mathcal{F}, u}(\epsilon)=\infty$. 
DEFINITION 2.10 The non-commutative foliated isoperimetric function is defined by

$$
\mathcal{I}_{\mathcal{F}, u}(\epsilon)=\frac{1}{D_{\mathcal{F}, u}(\epsilon)}
$$

The same ideas as used in the proof of Proposition 2.9 also establish:

PROPOSITION 2.11 Let $(V, \mathcal{F})$ be a $C^{1}$-foliation of a compact manifold $V$. Then for each $u \in K_{a f}^{1}(V, \mathcal{F})$, the class of $\mathcal{I}_{\mathcal{F}, u}(\epsilon)$ is a topological invariant.

Let us conclude this section with a discussion of profinite $K^{1}$-cocycles for $K_{a f}(M)$.

For each $N>0, U(N) \subset M(N, \mathbf{C}) \cong \mathbf{C}^{N^{2}}$ denotes the group of $N \times N$ unitary matrices considered as a subspace of the vector space of all matrices. Let $U(\infty)$ denote the stabilized super-group with the weak limit topology. The $C^{1}$-semi-norm of a $C^{1}$-function $g: \tilde{M} \rightarrow U(N)$ is defined as the supremum of the norms of the covariant derivatives of its matrix entries,

$$
\|g\|_{(1)}=\sup _{x \in \tilde{M}} \sup _{1 \leq k, \ell \leq N}\left\|\nabla g_{k \ell}\right\|_{x}
$$

DEFINITION 2.12 (Profinite $K^{1}-\Gamma$-cocycles) Let $\rho: \Lambda \rightarrow \Gamma$ be a submersion. A profinite $\Gamma$-cocycle for $M$ consists of the data $g^{p f}=\left\{\left(g_{i}, \Gamma_{i}, N_{i}\right) \mid 0 \leq i\right\}$ which satisfy:

1. $\Gamma_{i}$ is a finite quotient group of $\Gamma$, with $\Gamma_{0}=\Gamma$

2. $\pi_{i}: \tilde{M}_{i} \rightarrow M$ is the covering of $M$ associated to the surjection $\Lambda \rightarrow \Gamma \rightarrow \Gamma_{i}$

3. $g_{i}: \tilde{M}_{i} \rightarrow U\left(N_{i}\right)$ is a $C^{1}$ mapping with $\left\|g_{i}\right\|_{(1)}<1 / i$

4. For each $i \geq 0, \quad\left|\Gamma_{i}\right| \cdot\left[g_{i}\right]=\left[g_{0} \circ \pi_{i}\right] \in K^{1}\left(\tilde{M}_{i}\right)$.

$\left[g^{p f}\right] \in K^{1}(M)$ denotes the homotopy class of the stabilized map $g_{0} \rightarrow U\left(N_{0}\right) \subset$ $U(\infty)$.

Let $K_{\Gamma p f}^{1}(M) \subset K^{1}(M)$ denote the subset of classes represented by profinite $K^{1}$ - $\Gamma$-cocycles. When $\Gamma=\Lambda=\pi_{1}\left(M, x_{0}\right)$, then we simply write $K_{p f}^{1}(M)$.

PROPOSITION 2.13 $K_{\Gamma p f}^{1}(M)$ is a subgroup of $K_{\Gamma a f}^{1}(M)$.

Proof. The inverse of a profinite cocycle $g^{p f}=\left\{\left(g_{i}, \Gamma_{i}, N_{i}\right) \mid 0 \leq i\right\}$ is given by the obvious candidate $\left(g^{p f}\right)^{-1}=\left\{\left(g_{i}^{-1}, \Gamma_{i}, N_{i}\right) \mid 0 \leq i\right\}$. The sum operation is slightly more subtle: given $\left\{\left(g_{i}, \Gamma_{i}, N_{i}\right) \mid 0 \leq i\right\}$ and $\left\{\left(g_{i}^{\prime}, \Gamma_{i}^{\prime}, N_{i}^{\prime}\right) \mid 0 \leq i\right\}$, we define the "sum" $\left\{\left(g_{i}^{\prime \prime}, \Gamma_{i}^{\prime \prime}, N_{i}^{\prime \prime}\right) \mid 0 \leq i\right\}$ as follows:

For each $i \geq 0$ let $\Gamma_{i}^{\prime \prime}$ be the image of the "diagonal map" $\Gamma \rightarrow \Gamma_{i} \times \Gamma_{i}^{\prime}$. The covering $\tilde{M}_{i}^{\prime \prime} \rightarrow M$ associated to $\Gamma_{i}^{\prime \prime}$ has order at most $\left|\Gamma_{i}\right| \cdot\left|\Gamma_{i}^{\prime}\right|$. Then set

$$
g_{i}^{\prime \prime}: \tilde{M}_{i}^{\prime \prime} \longrightarrow \tilde{M}_{i} \times \tilde{M}_{i}^{\prime} \longrightarrow U\left(N_{i}\right) \times U\left(N_{i}^{\prime}\right) \subset U\left(N_{i}+N_{i}^{\prime}\right)
$$


which is the push-out product. The uniform pointwise norm of the gradient of $g_{i}^{\prime \prime}$ is at most that of the factors, so $\left\{\left(g_{i}^{\prime \prime}, \Gamma_{i}^{\prime \prime}, N_{i}^{\prime \prime}\right) \mid 0 \leq i\right\}$ is a profinite K-cocycle.

Note that the sum on the set $K_{\Gamma p f}^{1}(M)$ is induced by the operation $\left[g_{0}\right]+$ $\left[g_{0}^{\prime}\right]=\left[g_{0}^{\prime \prime}\right]$, which coincides with the standard sum operation on $K^{1}(M)$.

The most interesting part of the proposition is the assertion that the subgroup $K_{\Gamma p f}^{1}(M)$ is contained in $K_{\Gamma a f}^{1}(M)$. Fix $g^{p f}=\left\{\left(g_{i}, \Gamma_{i}, N_{i}\right) \mid 0 \leq i\right\}$. For each $i \geq 0$, there is a natural diffeomorphism of the finite covering $\tilde{M}_{i} \cong$ $\Gamma \backslash\left(\tilde{M}_{\Gamma} \times \Gamma_{i}\right)$, so that the unitary $g_{i}$ can be considered as a $\Gamma$-equivariant map $g_{i}: \tilde{M}_{\Gamma} \times \Gamma_{i} \rightarrow U\left(N_{i}\right)$, for the trivial action of $\Gamma$ on $U\left(N_{i}\right)$.

The space $\operatorname{Maps}\left(\Gamma_{i}, U\left(N_{i}\right)\right)$ is a compact group under pointwise multiplication, and acts naturally on the Hermitian vector space

$$
\tilde{\mathbf{E}}_{i}=\operatorname{Maps}\left(\Gamma_{i}, \mathbf{C}^{N_{i}}\right) \cong \mathbf{C}^{\left|\Gamma_{i}\right| \cdot N_{i}}
$$

by which $\operatorname{Maps}\left(\Gamma_{i}, U\left(N_{i}\right)\right) \subset U\left(\tilde{\mathbf{E}}_{i}\right)$. Then $g_{i}$ is equivalent by adjunction to a $\Gamma$-equivariant map $\tilde{g}_{i}: \tilde{M}_{\Gamma} \rightarrow \operatorname{Maps}\left(\Gamma_{i}, U\left(N_{i}\right)\right)$.

Define a foliated Hermitian flat bundle

$$
\mathbf{E}_{i}=\Gamma \backslash\left(\tilde{M}_{\Gamma} \times \tilde{\mathbf{E}}_{i}\right) \longrightarrow M
$$

with dimension $\left|\Gamma_{i}\right| \cdot N_{i}$. By the above discussion, $g_{i}$ determines a section $\hat{g}_{i} \in C^{1}\left(U\left(\mathbf{E}_{i}\right)\right)$, and $\left\|\hat{g}_{i}\right\|_{(1)} \leq\left\|g_{i}\right\|_{(1)}<1 / i$ where the left-hand-side is given by (2) and the middle term by (7).

The lift of the extended unitary $g_{0} \circ \pi_{i}: \tilde{M}_{i} \rightarrow U\left(N_{0}\right) \subset U\left(N_{i}\right)$ can also be "pushed-forward" to a fiberwise unitary automorphism $\hat{g}_{0, i} \in C^{1}\left(U\left(\mathbf{E}_{i}\right)\right)$. A homotopy $\left\{g_{t}\right\}$ between $\left|\Gamma_{i}\right| \cdot g_{i}: \tilde{M}_{i} \rightarrow U\left(\left|\Gamma_{i}\right| \cdot N_{i}\right)$ and $g_{0} \circ \pi_{i}: \tilde{M}_{i} \rightarrow$ $U\left(N_{0}\right) \subset U\left(\left|\Gamma_{i}\right| \cdot N_{i}\right)$ pushes forward to an admissible homotopy $\left\{\hat{g}_{t}\right\}$ between $\left|\Gamma_{i}\right| \cdot \hat{g}_{i}$ and $\hat{g}_{0, i}$. We leave it to the reader to check that the lift of $\hat{g}_{0, i}$ to $\pi^{*}\left(\hat{g}_{0, i}\right) \in C_{\mathcal{F}_{\Gamma}}^{1}\left(U\left(\pi^{!} \mathbf{E}_{i}\right)\right)$ is homotopic to $\pi^{*}\left(\left|\Gamma_{i}\right| \cdot \hat{g}_{0, i}\right) \in C_{\mathcal{F}_{\Gamma}}^{1}\left(U\left(\pi^{!} \mathbf{E}_{i}\right)\right)$. It follows that there is a homotopy $\left\{\hat{g}_{t}\right\}$ between $\pi^{*}\left(\hat{g}_{i}\right)$ and $\pi^{*}\left(\hat{g}_{i+1}\right)$ as required for an almost flat odd cocycle.

\section{Some foliation spectral analysis}

Fix a foliated measure space $V$ with foliation $\mathcal{F}$ having leaves of odd dimension $m$, and let $\mu$ be a transverse measure for $\mathcal{F}$ whose measure class is invariant under the transverse holonomy transformations. For each leaf $L \subset V$, we assume there is given a fixed Riemannian metric $\langle\cdot, \cdot\rangle_{L}$ on $T L$ such that $L$ becomes a complete manifold with bounded geometry; and the leafwise metrics, with the $C^{2}$-topology, vary bounded measurably with the transverse parameter. Recall that $\nabla^{L}$ is the associated Riemannian connection on the leaf $L$ with $\nabla^{\mathcal{F}}$ denoting the family of leafwise connections. The injectivity radius of $\mathcal{F}$ is the infimum of the injectivity radii of the leaves of $\mathcal{F}$, considered as complete Riemannian manifolds. 
For each leaf $L \subset V$, let $\mathcal{S}_{L} \rightarrow L$ denote the Clifford bundle of spinors associated to the Clifford algebra bundle $\mathcal{C}(T L)$, and let $\mathcal{D}_{L}: C_{c}^{\infty}\left(\mathcal{S}_{L}\right) \rightarrow C_{c}^{\infty}\left(\mathcal{S}_{L}\right)$ be the corresponding Dirac operator. $\mathcal{S} \rightarrow V$ denotes the union of the leafwise Spinor bundles, viewed as a (measurable) Hermitian bundle over $V$

Consider an Hermitian vector bundle $\mathbf{E}^{0} \rightarrow V$ of dimension $q$ whose restrictions $\mathbf{E}_{L}^{0} \rightarrow L$ to the leaves $L$ of $\mathcal{F}$ are smooth, and the curvature forms $\Omega_{L}$ of the leafwise Hermitian connections $\nabla \mathbf{E}_{L}^{0}$ are bounded with respect to the leafwise Riemannian metrics. We can then form the leafwise "geometric operators"

$$
\mathcal{D}_{L}=\mathcal{D}_{L} \otimes \nabla^{\mathbf{E}_{L}^{0}}
$$

defined on the (leafwise) compactly supported sections $C_{c}^{\infty}\left(\mathbf{E}_{L}\right)$ of the bundle $\mathbf{E}=\mathcal{S} \otimes \mathbf{E}^{0}$ restricted to the leaves of $\mathcal{F}$. The most natural definition of the operators in (8) is to form the leafwise Clifford bundles $\mathcal{S}_{L} \otimes E_{L}^{0} \rightarrow L$, and then declare $\mathcal{D}_{L}$ to be the associated "Dirac operator" (cf. Definition 2.4, [46]): At a point $x \in L$, choose an orthogonal framing $\left\{e_{1}, \ldots, e_{m}\right\}$ of $T_{x} L$ and extend these to local synchronous vector fields $\left\{\tilde{e}_{1}, \ldots, \tilde{e}_{m}\right\}$ about $x$ (cf. (1.29) of [46].) The Clifford algebra $\mathcal{C}\left(T_{x} L\right)$ is spanned by the monomials $\left\{e_{I}=\right.$ $\left.e_{i_{1}} \otimes \cdots \otimes e_{i_{p}} \mid I=\left(1_{1}<\cdots<i_{p}\right)\right\}$. Define $|I|=p$ for $I=\left(1_{1}<\cdots<i_{p}\right)$. Choose also a unitary framing $\left\{f_{1}, \ldots, f_{q}\right\}$ of $\mathbf{E}_{x}^{0}$ and extend to local $\nabla \mathbf{E}_{L_{-}}^{0}$ synchronous sections $\left\{\tilde{f}_{1}, \ldots, \tilde{f}_{q}\right\}$. Then for a general local section

$$
\begin{aligned}
s & =\sum_{\substack{|I| \leq m \\
1 \leq j \leq q}} s_{I, j} \cdot \tilde{e}_{I} \otimes \tilde{f}_{j} \quad \text { set } \\
\mathcal{D}_{L}(s)(x) & \left.\sum_{\substack{|I| \leq m \\
1 \leq \alpha \leq m \\
1 \leq j \leq q}} \nabla_{e_{\alpha}}^{L}\left(s_{\alpha, j}\right)\right|_{x} \cdot e_{\alpha} \otimes \tilde{e}_{I}(x) \otimes \tilde{f}_{j}(x)
\end{aligned}
$$

DEFINITION 3.1 $A$ foliation geometric operator $\mathcal{D}$ for $(V, \mathcal{F})$ is a collection of leafwise geometric operators $\left\{\mathcal{D}_{L} \mid L \subset V\right\}$ defined as in (8) for some leafwise Riemannian metric for $\mathcal{F}$ and some Hermitian vector bundle $\mathbf{E}^{0}$ as above.

We also introduce the algebra $\Psi \mathcal{D} \mathcal{O}_{c}^{0}(\mathbf{E}, \mathcal{F})$ of leafwise pseudo-differential operators of order $\leq 0$, acting on the leafwise sections $C_{c}^{\infty}\left(\mathbf{E}_{L}\right)$. This is defined in detail in (section A, Chapter 7 of [43]), with further properties of the leafwise pseudo-differential operators discussed in (section 3, [24]). Note that our definition of a leafwise pseudo-differential operator $\mathcal{P}=\left\{\mathcal{P}_{L} \mid L \subset V\right\} \in \Psi \mathcal{D} \mathcal{O}_{c}^{0}(\mathbf{E}, \mathcal{F})$ includes the important hypotheses that there is given a constant $\epsilon>0$ such that for each leaf $L$, the operator $\mathcal{P}_{L}$ is represented by a distributional kernel supported in an $\epsilon$-tube around the diagonal in the leaf $L$. Let $\epsilon(\mathcal{P})<\infty$ denote the least such $\epsilon$ so that this holds for every leaf $L$. The condition on supports for $\mathcal{P}$ implies that $\mathcal{P}_{L}: C_{c}^{\infty}\left(\mathbf{E}_{L}\right) \rightarrow C_{c}^{\infty}\left(\mathbf{E}_{L}\right)$.

DEFINITION 3.2 We say that a family $\tilde{\mathcal{D}}=\left\{\tilde{\mathcal{D}}_{L} \mid L \subset V\right\}$ of leafwise pseudo-differential operators is a compact perturbation of a geometric operator 
if there is a foliation geometric operator $\mathcal{D}$ acting on the leafwise sections of the bundle $\mathbf{E}=\mathcal{S} \otimes \mathbf{E}^{0}$, and a leafwise pseudo-differential operator $\mathcal{P} \in \Psi \mathcal{D} \mathcal{O}_{c}^{0}(\mathbf{E}, \mathcal{F})$ so that $\tilde{\mathcal{D}}_{L}=\mathcal{D}_{L}+\mathcal{P}_{L}$ for each leaf $L \subset V$ of $\mathcal{F}$.

We allow $V=M$ in this definition foliated by the single leaf $L=M$, and $\tilde{\mathcal{D}}_{M}=\mathcal{D}_{M}+\mathcal{P}_{M}$ with $\mathcal{P}_{M}$ a zero order pseudo-differential operator on $M$.

Introduce a constant which will appear repeatedly in the formulas that follow:

$$
\kappa(\tilde{\mathcal{D}})=\{1+\epsilon(\mathcal{P}) \cdot\|\mathcal{P}\|\}
$$

where $\|\mathcal{P}\|$ denotes the operator norm of $\mathcal{P}$.

We next establish an elementary estimate on the commutator of a compact perturbation of a geometric operator $\tilde{\mathcal{D}}$ with a unitary multiplier. This is the key estimate for the entire paper.

Let $\mathbf{E}^{1} \rightarrow V$ be a foliated Hermitian flat vector bundle of dimension $N$. Given $g \in C_{\mathcal{F}}^{1}\left(U\left(\mathbf{E}^{1}\right)\right)$, we define for each leaf $L$ a multiplier operator

$$
M\left(g_{L}\right): C_{c}^{1}\left(\mathbf{E}_{L} \otimes \mathbf{E}_{L}^{1}\right) \rightarrow C_{c}^{1}\left(\mathbf{E}_{L} \otimes \mathbf{E}_{L}^{1}\right)
$$

which acts by fiberwise multiplication with $g_{L}$ on the factor $\mathbf{E}_{L}^{1}$.

A leafwise geometric operator $\mathcal{D}_{L}$ on $C_{c}^{1}\left(\mathbf{E}_{L}\right)$ extends to the space of sections $C_{c}^{1}\left(\mathbf{E}_{L} \otimes \mathbf{E}_{L}^{1}\right)$ by means of a synchronous frame, exactly as for the coefficients $\mathbf{E}^{0}$. An operator $\mathcal{P} \in \Psi \mathcal{D} \mathcal{O}_{c}^{0}\left(\mathbf{E}^{L}, \mathcal{F}\right)$ such that $\epsilon(\mathcal{P})$ is less than the injectivity radius of the leaves has a similar extension, where the synchronous frame field is used to "freeze coefficients" for the auxiliary bundle $\mathbf{E}^{1}$ in a neighborhood of a point. Denote these extensions by $\tilde{\mathcal{D}}^{1}$ and $\mathcal{P}^{1}$ respectively.

Form the leafwise Hilbert space closures $\mathcal{H}\left(\mathbf{E}_{L} \otimes \mathbf{E}_{L}^{1}\right)$ of $C_{c}^{1}\left(\mathbf{E}_{L} \otimes \mathbf{E}_{L}^{1}\right)$ for each leaf $L$. For a family of bounded operators $\mathcal{A}_{L} \in \mathcal{B}\left(\mathcal{H}\left(\mathbf{E}_{L} \otimes \mathbf{E}_{L}^{1}\right)\right)$, let $\left\|\mathcal{A}_{L}\right\|$ denote the leafwise operator norms and set

$$
\|\mathcal{A}\|=\sup _{L \subset V}\left\|\mathcal{A}_{L}\right\|
$$

LEMMA 3.3 (Key estimate) Let $\tilde{\mathcal{D}}=\mathcal{D}+\mathcal{P}$ be a compact perturbation of a geometric operator acting on the sections of $\mathbf{E}=\mathcal{S} \otimes \mathbf{E}^{0}$ with the injectivity radius of $\mathcal{F}$ greater than $2 \epsilon(\mathcal{P})$. Let $g \in C_{\mathcal{F}}^{1}\left(U\left(\mathbf{E}^{1}\right)\right)$ be admissible for $\mathbf{E}_{L}^{1}$ a foliated Hermitian flat bundle. Then for each leaf $L$, the operator norm of the commutator of the extension $\tilde{\mathcal{D}}_{L}^{1}$ with the unitary multiplier $M\left(g_{L}\right)$ satisfies:

$$
\left\|\left[\tilde{\mathcal{D}}_{L}^{1}, M\left(g_{L}\right)\right]\right\| \leq\|g\|_{(1)} \cdot \kappa(\tilde{\mathcal{D}})
$$

Proof: The commutator of a geometric operator with a $C^{1}$-multiplier has the standard estimate

$$
\left\|\left[\mathcal{D}_{L}^{1}, M\left(g_{L}\right)\right]\right\| \leq\|g\|_{(1)} .
$$

so we need to estimate $\left\|\left[\mathcal{P}_{L}^{1}, M\left(g_{L}\right)\right]\right\|$. This will follow from explicit consideration of the action of $\mathcal{P}_{L}^{1}$ on sections. Choose a locally finite covering of $L$ by geodesic balls $\left\{B\left(x_{\alpha}, \epsilon^{\prime}\right)\right\}$ with centers $x_{\alpha} \in L$ and radius $\epsilon^{\prime}<\epsilon(\mathcal{P})$. Let $\left\{\lambda_{\alpha}\right\}$ 
be a partition-of-unity subordinate to this cover. Write $s \in C_{c}^{\infty}\left(\mathbf{E}_{L}\right)$ as a finite sum $s=\sum s_{\alpha}$ where $s_{\alpha}=\lambda_{\alpha} s$ has support contained in $B\left(x_{\alpha}, \epsilon^{\prime}\right)$. Fix synchronous orthonormal framings $\left\{\tilde{e}_{I}\right\}$ of $\mathcal{S}_{L},\left\{\tilde{f}_{1}, \ldots, \tilde{f}_{q}\right\}$ of $\mathbf{E}_{L}$, and $\left\{\tilde{h}_{1}, \ldots, \tilde{h}_{N}\right\}$ of $\mathbf{E}_{L}^{1}$ over $B\left(x_{\alpha}, 2 \epsilon(\mathcal{P})\right)$ with center at $x_{\alpha}$. Then the operator $\mathcal{P}_{L}^{1}$ acting on the sections of $\mathbf{E}_{L} \otimes \mathbf{E}_{L}^{1}$ supported in a disc $B\left(x_{\alpha}, \epsilon^{\prime}\right)$, expressed in these synchronous framings, is represented by a matrix whose entries are scalar-valued distributional kernels $k_{\alpha, i j}^{\mathcal{P}}\left(y, y^{\prime}\right)$ for $1 \leq i, j \leq q$. We estimate:

$$
\begin{aligned}
& \| {\left[\mathcal{P}_{L}^{1}, M\left(g_{L}\right)\right](s)\|=\|\left[\mathcal{P}_{L}^{1}, M\left(g_{L}\right)\right]\left(\sum \lambda_{\alpha} s_{I, j, \ell}^{\alpha} \tilde{e}_{I} \otimes \tilde{f}_{j} \otimes \tilde{h}_{\ell}\right) \| } \\
&= \| \sum \iint_{B\left(x_{\alpha}, 2 \epsilon(\mathcal{P})\right) \times B\left(x_{\alpha}, 2 \epsilon(\mathcal{P})\right)}\left\{\left(g_{L}\right)_{k \ell}(y) k_{\alpha, i j}^{\mathcal{P}}\left(y, y^{\prime}\right)-k_{\alpha, i j}^{\mathcal{P}}\left(y, y^{\prime}\right)\left(g_{L}\right)_{k \ell}\left(y^{\prime}\right)\right\} \\
& \cdot \lambda_{\alpha}\left(y^{\prime}\right) s_{I, j, \ell}^{\alpha}\left(y^{\prime}\right) \tilde{e}_{I} \otimes \tilde{f}_{j} \otimes \tilde{h}_{\ell} \| \\
& \leq \quad \sup _{\substack{y, y^{\prime} \in L \\
\left|y-y^{\prime}\right|<\epsilon(\mathcal{P})}}\left|\left(g_{L}\right)_{k \ell}\left(y^{\prime}\right)-\left(g_{L}\right)_{k \ell}(y)\right| \cdot\left\|\mathcal{P}_{L}\right\| \cdot\|s\| \\
& \leq \quad\|g\|_{(1)} \cdot \epsilon(\mathcal{P}) \cdot\left\|\mathcal{P}_{L}\right\| \cdot\|s\|
\end{aligned}
$$

where the estimate (14) follows by noting that the frame field $\left\{\tilde{h}_{1}, \ldots, \tilde{h}_{N}\right\}$ of $\mathbf{E}^{1}$ over $B\left(x_{\alpha}, 2 \epsilon(\mathcal{P})\right)$ is synchronous at each point as $\mathbf{E}_{L}^{1}$ is flat, and then applying elementary calculus to the difference term in (13).

Assume that $\tilde{\mathcal{D}}_{L}^{1}$ is essentially self-adjoint on $\mathcal{H}\left(\mathbf{E}_{L} \otimes \mathbf{E}_{L}^{1}\right)$. Let $d \sigma\left(\tilde{\mathcal{D}}_{L}^{1}\right)(\lambda)$ denote the spectral measure for $\tilde{\mathcal{D}}_{L}^{1}$, and introduce the (leafwise) spectral projections

$$
\chi_{\left[\lambda_{0}, \lambda_{1}\right]}\left(\tilde{\mathcal{D}}_{L}^{1}\right)=\int_{\lambda_{0}}^{\lambda_{1}} d \sigma\left(\tilde{\mathcal{D}}_{L}^{1}\right) \in \mathcal{B}\left(\mathcal{H}\left(\mathbf{E}_{L} \otimes \mathbf{E}_{L}^{1}\right)\right)
$$

where $-\infty \leq \lambda_{0}<\lambda_{1} \leq \infty$. Lemma 3.3 is the first step in the following key estimate:

PROPOSITION 3.4 Let $\tilde{\mathcal{D}}$ be a compact perturbation of a geometric operator, and $g \in C_{\mathcal{F}}^{1}\left(U\left(\mathbf{E}^{1}\right)\right)$ be admissible with $\mathbf{E}_{L}^{1}$ Hermitian flat for each leaf $L$. For $\lambda \in \mathbf{R}$ let $\Pi_{L}(\lambda)(g)$ be the projection onto the closed Hilbert subspace

$$
\operatorname{Range}\left(\chi_{[\lambda, \infty)}\left(\tilde{\mathcal{D}}_{L}^{1}\right)\right) \cap \operatorname{Range}\left(M\left(g_{L}^{*}\right) \circ \chi_{(-\infty, \lambda]}\left(\tilde{\mathcal{D}}_{L}^{1}\right)\right)
$$

Then for all $\epsilon>0$ there is the following estimate on the operator norms

$$
\left\|\chi_{[\lambda+\epsilon, \infty)}\left(\tilde{\mathcal{D}}_{L}^{1}\right) \circ \Pi_{L}(\lambda)(g)\right\| \leq \sqrt{\|g\|_{(1)} \cdot \kappa(\tilde{\mathcal{D}}) / \epsilon}
$$


Proof: First note that

$$
\begin{aligned}
\operatorname{Range}\left(M\left(g_{L}^{*}\right) \circ \chi_{(-\infty, \lambda]}\left(\tilde{\mathcal{D}}_{L}^{1}\right)\right) & =\operatorname{Range}\left(M\left(g_{L}^{*}\right) \circ \chi_{(-\infty, \lambda]}\left(\tilde{\mathcal{D}}_{L}^{1}\right) \circ M\left(g_{L}\right)\right) \\
& =\operatorname{Range}\left(\chi_{(-\infty, \lambda]}\left(M\left(g_{L}^{*}\right) \circ \tilde{\mathcal{D}}_{L}^{1} \circ M\left(g_{L}\right)\right)\right)
\end{aligned}
$$

Set $K=\|g\|_{(1)} \cdot \kappa(\tilde{\mathcal{D}})$, then Lemma 3.3 yields the estimate

$$
\left\|\tilde{\mathcal{D}}_{L}^{1}-M\left(g_{L}^{*}\right) \circ \tilde{\mathcal{D}}_{L}^{1} \circ M\left(g_{L}\right)\right\| \leq K
$$

Since

$$
\chi_{[\lambda+\epsilon, \infty)}\left(\tilde{\mathcal{D}}_{L}^{1}\right) \circ \Pi_{L}(\lambda)(g)=\chi_{[\lambda+\epsilon, \infty)}\left(\tilde{\mathcal{D}}_{L}^{1}\right) \circ \Pi_{L}(\lambda)(g) \circ \Pi_{L}(\lambda)(g)
$$

it suffices to estimate the norm of $\chi_{[\lambda+\epsilon, \infty)}\left(\tilde{\mathcal{D}}_{L}^{1}\right) \circ \Pi_{L}(\lambda)(g)$ restricted to the range of $\Pi_{L}(\lambda)(g)$. Let $\phi \in$ Range $\left(\Pi_{L}(\lambda)(g)\right)$ have norm 1 , and write $\phi=\phi_{1}+\phi_{2}$ where

$$
\begin{aligned}
& \phi_{1}=\chi_{[\lambda, \lambda+\epsilon)}\left(\tilde{\mathcal{D}}_{L}^{1}\right) \phi \\
& \phi_{2}=\chi_{[\lambda+\epsilon, \infty)}\left(\tilde{\mathcal{D}}_{L}^{1}\right) \phi
\end{aligned}
$$

so that we must estimate $\left\|\phi_{2}\right\|$. Use (18), Cauchy-Schwartz inequality, the spectral theorem, orthogonality of the ranges of $\chi_{[\lambda, \lambda+\epsilon)}\left(\tilde{\mathcal{D}}_{L}^{1}\right)$ and $\chi_{[\lambda+\epsilon, \infty)}\left(\tilde{\mathcal{D}}_{L}^{1}\right)$, and their invariance under $\tilde{\mathcal{D}}_{L}^{1}$ to deduce

$$
\begin{aligned}
K & \geq\left\langle\left(\tilde{\mathcal{D}}_{L}^{1}-M\left(g_{L}^{*}\right) \circ \tilde{\mathcal{D}}_{L}^{1} \circ M\left(g_{L}\right)\right) \phi, \phi\right\rangle \\
& \geq\left\langle\left(\tilde{\mathcal{D}}_{L}^{1}-\lambda\right) \phi, \phi\right\rangle \\
& =\left\langle\left(\tilde{\mathcal{D}}_{L}^{1}-\lambda\right) \phi_{1}, \phi_{1}\right\rangle+\left\langle\left(\tilde{\mathcal{D}}_{L}^{1}-\lambda\right) \phi_{2}, \phi_{2}\right\rangle \\
& \geq\left\langle\left(\tilde{\mathcal{D}}_{L}^{1}-\lambda\right) \phi_{2}, \phi_{2}\right\rangle \\
& \geq \epsilon \cdot\left\langle\phi_{2}, \phi_{2}\right\rangle
\end{aligned}
$$

which yields (17).

\section{Estimates for the odd foliation index}

In this section, we construct the index data for a foliated space $(V, \mathcal{F})$ with an invariant transverse measure $\mu$, a leafwise essentially self-adjoint operator $\tilde{\mathcal{D}}$ which is a compact perturbation of a geometric operator, and $u \in K_{a f}^{1}(V, \mathcal{F})$ represented by $g \in C_{\mathcal{F}}^{1}\left(U\left(\mathbf{E}^{1}\right)\right)$ for $\mathbf{E}^{1}$ a foliated Hermitian flat vector bundle. The "index theory" for self-adjoint operators along foliations, as used in this section, is developed in detail in the papers (section 5, [15]; Appendix A, [24]; [25].)

Extend $\tilde{\mathcal{D}}$ to the operator $\tilde{\mathcal{D}}^{1}$ on $C_{c}^{1}\left(\mathbf{E} \otimes \mathbf{E}^{1}\right)$ and define $M\left(g_{L}\right)$ as in the last section. Let $\lambda: V \rightarrow \mathbf{R}$ be a Borel function which is constant on the leaves 
of $\mathcal{F}$. We write $\lambda(L)$ for the value of $\lambda(x)$ at any point $x \in L$. For each leaf $L$ construct the spectral projections:

$$
\begin{aligned}
P_{L}^{+}(\lambda) & =\chi_{[\lambda(L), \infty)}\left(\tilde{\mathcal{D}}_{L}\right) \in \mathcal{B}\left(\mathcal{H}\left(\mathbf{E}_{L}\right)\right) \\
P_{L}^{1+}(\lambda) & =\chi_{[\lambda(L), \infty)}\left(\tilde{\mathcal{D}}_{L}^{1}\right) \in \mathcal{B}\left(\mathcal{H}\left(\mathbf{E}_{L} \otimes \mathbf{E}_{L}^{1}\right)\right)
\end{aligned}
$$

and the leafwise " $\lambda$-Toeplitz compressions" of the unitary multipliers $M\left(g_{L}\right)$

$$
T_{L}^{+}(\lambda)\left(g_{L}\right)=P_{L}^{1+}(\lambda) \circ M\left(g_{L}\right) \circ P_{L}^{1+}(\lambda)
$$

The range of the operator $P_{L}^{1+}(\lambda)$ is a closed Hilbert subspace $\mathcal{H}_{L}^{+}(\lambda) \subset$ $\mathcal{H}\left(\mathbf{E}_{L} \otimes \mathbf{E}_{L}^{1}\right)$. The correspondence $L \mapsto \mathcal{H}_{L}^{+}(\lambda)$ forms a measurable field of Hilbert spaces over the foliated space $(V, \mathcal{F})$ in the sense of Connes [12, 13]. Introduce the foliation von Neumann algebra $W_{\mu}^{*}(V, \mathcal{F})$ associated with the transverse measure class of $\mu$; then there is the basic fact from [25]:

PROPOSITION 4.1 Suppose that $g \in U_{\mathcal{F}}^{1}\left(\mathbf{E}^{1}\right)$ with $\|g\|_{(1)}<\infty$. Then the family of operators $\left\{T_{L}^{+}(\lambda)\left(g_{L}\right)\right\}=\left\{T_{L}^{+}(\lambda)\left(g_{L}\right) \mid L \subset V\right\}$, where $T_{L}^{+}(\lambda)\left(g_{L}\right) \in$ $\mathcal{B}\left(\mathcal{H}_{L}^{+}(\lambda)\right)$, is Fredholm in the sense of Breuer $[6,7]$. The von Neumann index of $\left\{T_{L}^{+}(\lambda)\left(g_{L}\right)\right\}$ is the $K_{0}$-class

$$
\mathcal{I}(\tilde{\mathcal{D}}, g)=\left[\left\{\mathcal{I}_{L}^{+}(\lambda)(g)\right\}\right]-\left[\left\{\mathcal{I}_{L}^{-}(\lambda)(g)\right\}\right] \in K_{0}\left(W_{\mu}^{*}(V, \mathcal{F})\right)
$$

where for each leaf $L$

$$
\begin{aligned}
& \mathcal{I}_{L}^{+}(\lambda)(g)=\Pi\left(\operatorname{ker}\left\{T_{L}^{+}(\lambda)\left(g_{L}\right)\right\}\right) \\
& \mathcal{I}_{L}^{-}(\lambda)(g)=\Pi\left(\operatorname{ker}\left\{T_{L}^{+}(\lambda)\left(g_{L}^{*}\right)\right\}\right)
\end{aligned}
$$

are the leafwise projection operators onto the the kernels of $T_{L}^{+}(\lambda)\left(g_{L}\right)$ and $T_{L}^{+}(\lambda)\left(g_{L}^{*}\right)$, respectively.

Let us now assume that the transverse measure $\mu$ for $\mathcal{F}$ is invariant under the leafwise holonomy transformations, so that it determines a trace $\operatorname{tr}_{\mu}$ on the algebra $W_{\mu}^{*}(V, \mathcal{F})$ and induces a homomorphism $T_{\mu}: K_{0}\left(W_{\mu}^{*}(V, \mathcal{F})\right) \rightarrow \mathbf{R}$. A basic observation of Connes is that the family of leafwise operators $\left\{\mathcal{I}_{L}^{ \pm}(\lambda)(g)\right\}$ obtained from leafwise elliptic pseudo-differential operators are in the domain of the trace $t r_{\mu}$ for any invariant transverse measure for $\mathcal{F}$. The analytic $\mu$-index of the family of leafwise Toeplitz operators $\left\{T_{L}^{+}(\lambda)\left(g_{L}\right)\right\}$ is defined as

$$
T_{\mu}(\mathcal{I}(\tilde{\mathcal{D}}, g))=\operatorname{tr}_{\mu}\left(\left\{\mathcal{I}_{L}^{+}(\lambda)(g)\right\}\right)-\operatorname{tr}_{\mu}\left(\left\{\mathcal{I}_{L}^{-}(\lambda)(g)\right\}\right)
$$

The measured foliation index theorem of Connes $[13,14]$ yields a topological formula for $T_{\mu}(\mathcal{I}(\tilde{\mathcal{D}}, g))$. Let $\sigma^{+}\left(\tilde{\mathcal{D}}^{1}, g\right): S \mathcal{F} \rightarrow \operatorname{Aut}\left(\mathbf{E} \otimes \mathbf{E}^{1}\right)$ denote the symbol of the compression of $M(g)$ to the fiberwise positive eigenspace for the symbol of $P^{+}(\lambda)$, where $S \mathcal{F}$ is the unit cosphere bundle over $V$ consisting of unit cotangent vectors to the leaves of $\mathcal{F}$. Let $\operatorname{Td}(\mathcal{F})$ denote the Todd class of $T \mathcal{F}$, and $\left[C_{\mu}\right]$ the Ruelle-Sullivan class of $\mu$. Let $\psi: H_{\mathcal{F}}^{*}(V) \rightarrow H_{\mathcal{F}}^{*+m-1}(S \mathcal{F})$ denote the Thom isomorphism for the leafwise deRham cohomology of $\mathcal{F}$. The index theorem for Toeplitz compression operators along leaves (Appendix B, [24]; [25]) then states: 


\section{THEOREM 4.2 (Odd index theorem for foliations)}

$$
T_{\mu}(\mathcal{I}(\tilde{\mathcal{D}}, g))=(-1)^{m}\left\langle\psi^{-1}\left(c h^{*}\left(\sigma^{+}\left(\tilde{\mathcal{D}}^{1}, g\right)\right)\right) \cup T d(\mathcal{F}),\left[C_{\mu}\right]\right\rangle .
$$

We can now prove the main technical result of this paper:

THEOREM 4.3 (Foliation spectral estimate) Let $\tilde{\mathcal{D}}=\mathcal{D}+\mathcal{P}$ be a compact perturbation of a geometric operator, and $g \in C_{\mathcal{F}}^{1}\left(U\left(\mathbf{E}^{1}\right)\right)$ admissible. Set $K=\|g\|_{(1)} \cdot \kappa(\tilde{\mathcal{D}})$. For any $\lambda \in \mathbf{R}$ and $\epsilon>K$

$$
\left|T_{\mu}(\mathcal{I}(\tilde{\mathcal{D}}, g))\right| \leq \frac{2}{1-\sqrt{K / \epsilon}} \cdot \operatorname{tr}_{\mu}\left(\left\{\chi_{[\lambda(L), \lambda(L)+\epsilon)}\left(\tilde{\mathcal{D}}^{1}\right)\right\}\right)
$$

Proof: Fix a leaf $L$. Observe that the range of $\mathcal{I}_{L}^{+}(\lambda)(g)$ is contained in the closed Hilbert space (16), which is the range of the leafwise projection operator $\Pi_{L}(\lambda)(g)$. Thus, we have the operator estimates

$$
\mathcal{I}_{L}^{+}(\lambda)(g) \leq \Pi_{L}(\lambda)(g)
$$

Apply Proposition 3.4 to obtain

$$
\begin{aligned}
\operatorname{tr}_{\mu}\left(P_{L}^{+}(\lambda+\epsilon) \circ \Pi_{L}(\lambda)(g)\right) & =\operatorname{tr}_{\mu}\left(P_{L}^{+}(\lambda+\epsilon) \circ \Pi_{L}(\lambda)(g) \circ \Pi_{L}(\lambda)(g)\right) \\
& \leq \sqrt{K / \epsilon} \cdot \operatorname{tr}_{\mu}\left(\Pi_{L}(\lambda)(g)\right)
\end{aligned}
$$

Then by positivity of the trace $t_{\mu}$ and the estimate (26) we have

$$
\begin{aligned}
\operatorname{tr}_{\mu}\left(\mathcal{I}^{+}(\lambda)(g)\right) \leq & \operatorname{tr}_{\mu}\left(\left\{\Pi_{L}(\lambda)(g)\right\}\right) \\
= & \operatorname{tr}_{\mu}\left(\left\{\chi_{[\lambda(L), \lambda(L)+\epsilon)}\left(\tilde{\mathcal{D}}_{L}^{1}\right)+P_{L}^{+}(\lambda+\epsilon)\right\} \circ \Pi_{L}(\lambda)(g)\right) \\
= & \operatorname{tr}_{\mu}\left(\left\{\chi_{[\lambda(L), \lambda(L)+\epsilon)}\left(\tilde{\mathcal{D}}_{L}^{1}\right) \circ \Pi_{L}(\lambda)(g)\right\}\right) \\
& +\operatorname{tr}_{\mu}\left(\left\{P_{L}^{+}(\lambda+\epsilon) \circ \Pi_{L}(\lambda)(g)\right\}\right) \\
\leq & \operatorname{tr}_{\mu}\left(\left\{\chi_{[\lambda(L), \lambda(L)+\epsilon)}\left(\tilde{\mathcal{D}}_{L}^{1}\right) \circ \Pi(\lambda)(g)\right\}\right) \\
& +\sqrt{K / \epsilon} \cdot \operatorname{tr}_{\mu}\left(\left\{\Pi_{L}(\lambda)(g)\right\}\right) \\
\leq & \operatorname{tr}_{\mu}\left(\left\{\chi_{[\lambda(L), \lambda(L)+\epsilon)}\left(\tilde{\mathcal{D}}_{L}^{1}\right)\right\}\right)+\sqrt{K / \epsilon} \cdot \operatorname{tr}_{\mu}\left(\left\{\Pi_{L}(\lambda)(g)\right\}\right)
\end{aligned}
$$

Thus,

$$
\begin{aligned}
(1-\sqrt{K / \epsilon}) \cdot \operatorname{tr}_{\mu}\left(\left\{\mathcal{I}_{L}^{+}(\lambda)(g)\right\}\right) & \leq(1-\sqrt{K / \epsilon}) \cdot \operatorname{tr}_{\mu}\left(\left\{\Pi_{L}(\lambda)(g)\right\}\right) \\
& \leq \operatorname{tr}_{\mu}\left(\left\{\chi_{[\lambda(L), \lambda(L)+\epsilon)}\left(\tilde{\mathcal{D}}_{L}^{1}\right)\right\}\right)
\end{aligned}
$$

Replacing $M\left(g_{L}^{*}\right)$ with $M\left(g_{L}\right)$ we get a similar estimate for $\operatorname{tr}_{\mu}\left(\left\{\mathcal{I}_{L}^{+}(\lambda)(g)\right\}\right)$, and (25) follows by combining these two estimates. 
COROLLARY 4.4 Let $\tilde{\mathcal{D}}=\mathcal{D}+\mathcal{P}$ be a compact perturbation of a geometric operator, and $g \in C_{\mathcal{F}}^{1}\left(U\left(\mathbf{E}^{1}\right)\right)$ admissible. Suppose there exists $\epsilon>\|g\|_{(1)} \cdot \kappa(\tilde{\mathcal{D}})$ and a Borel function $\lambda: V \rightarrow \mathbf{R}$ which is $\mu$-almost everywhere constant on leaves of $\mathcal{F}$, such that $\chi_{[\lambda(L), \lambda(L)+\epsilon)}\left(\tilde{\mathcal{D}}_{L}\right)=0$ for $\mu$-almost every leaf $L \subset V$. Then $T_{\mu}(\mathcal{I}(\tilde{\mathcal{D}}, g))=0$.

COROLLARY 4.5 (Foliated isoperimetric estimate) Let $(V, \mathcal{F}, \mu)$ be a measured foliation endowed with a leafwise Riemannian metric, and leaves of odd dimension $m$. Suppose that $L$ is simply connected for $\mu$-almost every leaf $L \subset V$. Let $\tilde{\mathcal{D}}=\mathcal{D}+\mathcal{P}$ be a compact perturbation of a foliation geometric operator. Then for each $u \in K_{a f}^{1}(V, \mathcal{F}), \epsilon>0$ and Borel function $\lambda: V \rightarrow \mathbf{R}$ which is $\mu$-almost everywhere constant on leaves of $\mathcal{F}$,

$$
\begin{aligned}
& \operatorname{tr}_{\mu}\left(\left\{\chi_{[\lambda(L), \lambda(L)+\epsilon)}\left(\tilde{\mathcal{D}}_{L}\right)\right\}\right) \geq \\
& \quad \frac{1}{4}\left|\left\langle\psi^{-1}\left(\operatorname{ch}^{*}\left(\sigma^{+}\left(\tilde{\mathcal{D}}^{1}, g\right)\right)\right) \cup \operatorname{Td}(\mathcal{F}),\left[C_{\mu}\right]\right\rangle\right| \cdot \mathcal{I}_{\mathcal{F}, u}(\epsilon / 4 \kappa(\tilde{\mathcal{D}}))
\end{aligned}
$$

where $g \in C_{\mathcal{F}}^{1}\left(U\left(\mathbf{E}^{1}\right)\right)$ represents $u \in K_{a f}^{1}(V, \mathcal{F})$.

Proof. Fix $\epsilon>0$. Choose a foliated Hermitian flat bundle $\mathbf{E}^{1}$ with minimum dimension $N_{\epsilon}=D_{\mathcal{F}, u}(\epsilon)$ so that $u$ is represented by $g_{\epsilon} \in C_{\mathcal{F}}^{1}\left(U\left(\mathbf{E}_{\epsilon}\right)\right)$ with $\left\|g_{\epsilon}\right\|_{(1)}<\epsilon / 4 \kappa(\tilde{\mathcal{D}})$. Then apply Theorem 4.3 and the Foliation Index Theorem 4.2 to obtain

$$
\begin{aligned}
4 \cdot \operatorname{tr}_{\mu}\left(\left\{\chi_{[\lambda(L), \lambda(L)+\epsilon)}\left(\tilde{\mathcal{D}}_{L}^{1}\right)\right\}\right) & \geq \frac{2 \cdot \operatorname{tr}_{\mu}\left(\left\{\chi_{[\lambda(L), \lambda(L)+\epsilon)}\left(\tilde{\mathcal{D}}_{L}^{1}\right)\right\}\right)}{1-\sqrt{\kappa(\tilde{\mathcal{D}})\left\|g_{\epsilon}\right\|_{(1)} / \epsilon}} \\
& \geq\left|\left\langle\psi^{-1}\left(\operatorname{ch}^{*}\left(\sigma^{+}\left(\tilde{\mathcal{D}}^{1}, g\right)\right)\right) \cup \operatorname{Td}(\mathcal{F}),\left[C_{\mu}\right]\right\rangle\right|
\end{aligned}
$$

It is given that the fundamental group of $\mu$-almost every leaf $L$ is trivial. For such a leaf, the holonomy transport defines an Hermitian isomorphism of flat bundles, $U_{L}: \mathbf{E}_{L}^{1} \cong L \times \mathbf{C}^{N_{\epsilon}}$, between the restricted bundle $\mathbf{E}_{L}^{1} \rightarrow L$ and the trivial bundle. The holonomy transport depends continuously on the initial parameter, so if we let $L_{x}$ denote the leaf of $\mathcal{F}$ through $x \in V$, then the correspondence $x \mapsto U_{L_{x}}$ is a $\mu$-measurable family of unitary maps. Each map $U_{L}$ induces an isomorphism between leafwise Hilbert spaces

$$
\tilde{U}_{L}: \mathcal{H}\left(\mathbf{E}_{L} \otimes \mathbf{E}_{L}^{1}\right) \cong \mathcal{H}\left(\mathbf{E}_{L} \otimes \mathbf{C}^{N_{\epsilon}}\right)
$$

The family of maps $x \mapsto U_{L_{x}}$ defines a unitary in the foliation von Neumann algebra $W_{\mu}^{*}(V, \mathcal{F})$ associated to the measure $\mu$. By the definition of the extension $\mathcal{D}_{L}^{1}$, the Hermitian isomorphism $\tilde{U}_{L}$ conjugates $\mathcal{D}_{L}^{1}$ with the "diagonal" extension of $\mathcal{D}_{L}$ to $N_{\epsilon}$-copies of itself. Therefore, the spectral projection $\chi_{[\lambda, \lambda+\epsilon]}\left(\tilde{\mathcal{D}}^{1}\right)$ is unitarily conjugate to $N_{\epsilon}$-copies of the the spectral projection $\chi_{[\lambda, \lambda+\epsilon]}(\tilde{\mathcal{D}})$, which implies

$$
\operatorname{tr}_{\mu}\left(\left\{\chi_{[\lambda(L), \lambda(L)+\epsilon)}\left(\tilde{\mathcal{D}}_{L}^{1}\right)\right\}\right)=N_{\epsilon} \cdot \operatorname{tr}_{\mu}\left(\left\{\chi_{[\lambda(L), \lambda(L)+\epsilon)}\left(\tilde{\mathcal{D}}_{L}\right)\right\}\right)
$$


Combining this last estimate with (28) and dividing through by $4 N_{\epsilon}$ yields (27).

REMARK 4.6 There is yet another way to define "almost flat odd K-theory", which allows for unitary automorphisms of Hermitian bundles which are "almost flat". This definition has the advantage that there is a natural module action of the even almost flat theory on the odd almost flat theory. We have avoided this possibly more general definition, due to a point which the reader may have noted in the above proof. It is critical for our calculation of the spectral density that there exists a global unitary between the Hilbert spaces $\mathcal{H}\left(\mathbf{E}_{L} \otimes \mathbf{E}_{L}^{1}\right)$ and $\mathcal{H}\left(\mathbf{E}_{L} \otimes \mathbf{C}^{N_{\epsilon}}\right)$ which depends measurably on the leaf. It is not clear how to construct such isomorphisms for almost flat bundles.

\section{Applications to coverings}

In this section, we derive applications of Theorem 4.3 to the compact perturbations of a $\Gamma$-periodic geometric operator on a normal covering $M_{\Gamma} \rightarrow M$.

Baum and Douglas define a Chern character on K-homology [3, 4] which for the odd K-theory of $M$ is a homomorphism $c h_{*}: K_{1}(M) \rightarrow H_{o d d}(M ; \mathbf{R})$. This map is characterized by an evaluation property: for $\mathcal{D}_{M}$ a symmetric elliptic operator on $M$ with K-homology class $\left[\mathcal{D}_{M}\right] \in K_{1}(M)$ and $g: M \rightarrow U(N)$ a continuous unitary with K-cohomology class $u \in K^{1}(M)$,

$$
\left\langle c h^{*}(u), c h_{*}\left(\left[\mathcal{D}_{M}\right]\right)\right\rangle=\operatorname{Index}\left\{\chi_{[0, \infty)}\left(\mathcal{D}_{M}\right) \circ M(g) \circ \chi_{[0, \infty)}\left(\mathcal{D}_{M}\right)\right\} \in \mathbf{Z}
$$

THEOREM 5.1 (No gaps) Suppose that there is $u \in K_{\Gamma a f}^{1}(M) \subset K^{1}(M)$ such that

$$
\left\langle c h^{*}(u), c h_{*}\left(\left[\mathcal{D}_{M}\right]\right)\right\rangle=(-1)^{m}\left\langle\psi^{-1}\left(\operatorname{ch}^{*}\left(\sigma^{+}\left(\mathcal{D}_{M}^{1}, g\right)\right)\right) \cup \operatorname{Td}(M),[M]\right\rangle \neq 0 .
$$

1. Let $\tilde{\mathcal{D}}_{\Gamma}: C_{c}^{1}\left(\mathbf{E}_{\Gamma}\right) \rightarrow C_{c}^{1}\left(\mathbf{E}_{\Gamma}\right)$ be the lift to $\tilde{M}_{\Gamma}$ of a pseudo-differential operator $\tilde{\mathcal{D}}_{M}$ on $C^{\infty}\left(\mathbf{E}_{M}\right)$ which is a compact perturbation of $\mathcal{D}_{M}$, then $\sigma\left(\tilde{\mathcal{D}}_{\Gamma}\right)=\mathbf{R}$.

2. Let $\tilde{\mathcal{D}}$ be a compact perturbation of the leafwise operator $\mathcal{D}_{\Gamma}$ obtained from the lift of $\mathcal{D}_{M}$ to the foliation $\mathcal{F}_{\Gamma}$ of $V_{\Gamma}$. Then for $\mu_{\Gamma}$-almost every leaf $L$ of $\mathcal{F}_{\Gamma}$, the spectrum $\sigma\left(\tilde{\mathcal{D}}_{L}\right)=\mathbf{R}$.

REMARK. Note that in the first case (5.1.1) the compact perturbation $\tilde{\mathcal{D}}_{\Gamma}$ is $\Gamma$-periodic on $\tilde{M}_{\Gamma}$, while for the second case $(5.1 .2)$ we allow for a $\Gamma$-random compact perturbation within the context of the foliation $\mathcal{F}_{\Gamma}$. In the latter case, the the foliation index theorem technique only yields that $\sigma\left(\tilde{\mathcal{D}}_{L}\right)=\mathbf{R}$ for almost all perturbations.

Proof of Theorem 5.1: Introduce the foliated space $\left(V_{\Gamma}, \mathcal{F}_{\Gamma}\right)$, and lift $\mathcal{D}_{M}$ to a leafwise operator $\mathcal{D}_{\Gamma}$ for $\mathcal{F}_{\Gamma}$. $\tilde{\mathcal{D}}$ will denote a compact perturbation of $\mathcal{D}_{\Gamma}$. 
The case (5.1.1) assumes that $\tilde{\mathcal{D}}$ is a lift of a pseudo-differential operator on $M$, hence the restriction $\tilde{\mathcal{D}}_{L}=\tilde{\mathcal{D}}_{\Gamma}$ for each leaf $L$, and thus (5.1.1) follows from (5.1.2).

Observe that the measure space $X_{\Gamma} \cong\left\{y_{0}\right\} \times X_{\Gamma} \rightarrow V_{\Gamma}$ is a complete transversal for $\mathcal{F}$, so we can (non-uniquely) parametrize the leaves of $\mathcal{F}_{\Gamma}$ by the points of this space. Let $L_{x}$ denote the leaf through $x \in X_{\Gamma}$.

LEMMA 5.2 The spectral projection operators $x \mapsto \chi_{\left[\lambda_{0}, \lambda_{1}\right]}\left(\tilde{\mathcal{D}}_{L_{x}}\right)$ are measurable functions for the operator topology on $\mathcal{B}\left(\mathcal{H}_{L_{x}}\right)$ where $\mathcal{H}_{L_{x}} \cong \mathcal{H}_{\Gamma}$ is the Hilbert space closure of $C_{c}^{1}\left(\mathbf{E}_{\Gamma}\right)$.

Proof: The characteristic function $\chi_{\left[\lambda_{0}, \lambda_{1}\right]}(\xi)$ is the limit of continuous functions $\varphi_{t} \rightarrow \chi_{\left[\lambda_{0}, \lambda_{1}\right]}$ for $t \rightarrow 0$ and each $0 \leq \varphi_{t}(\xi) \leq 1$ with $\operatorname{support}\left(\varphi_{t}\right) \subset[-1,2]$. Thus, the spectral projection $\chi_{\left[\lambda_{0}, \lambda_{1}\right]}\left(\tilde{\mathcal{D}}_{L_{x}}\right)$ is the limit in the operator topology of the sequence of operators $\varphi_{t}\left(\tilde{\mathcal{D}}_{L_{x}}\right)$, and the convergence is uniform in $x$. The spectral theorem implies that $\varphi_{t}\left(\tilde{\mathcal{D}}_{L_{x}}\right)$, for all $t>0$, depends continuously on $x$ (cf. Proposition 5.8, [46]) and hence the limit $\chi_{\left[\lambda_{0}, \lambda_{1}\right]}\left(\tilde{\mathcal{D}}_{L_{x}}\right)$ depends measurably on $x$.

Suppose that there exists a set of positive $\tilde{\mu}$-measure $Z \subset X_{\Gamma}$ such that for each $x \in Z$, the spectrum $\sigma\left(\tilde{\mathcal{D}}_{L_{x}}\right) \neq \mathbf{R}$. Countable additivity of the measure $\tilde{\mu}$ implies that there exists $\lambda \in \mathbf{R}, \epsilon>0$ and a $\tilde{\mu}$-measurable subset $Z_{\epsilon} \subset Z$ of positive $\tilde{\mu}$-measure so that $\chi_{[\lambda, \lambda+\epsilon]}\left(\tilde{\mathcal{D}}_{L_{x}}\right)=0$ for all $x \in Z_{\epsilon}$.

Note that if $x, x^{\prime} \in X_{\Gamma}$ are $\Gamma$-equivalent - that is, there is $\gamma \in \Gamma$ such that $\gamma x=x^{\prime}$ - then the leaves $L_{x}$ and $L_{x^{\prime}}$ are the same for $\mathcal{F}_{\Gamma}$, and hence $\sigma\left(\tilde{\mathcal{D}}_{L_{x}}\right)=\sigma\left(\tilde{\mathcal{D}}_{L_{x^{\prime}}}\right)$. We can therefore assume that $Z_{\epsilon}$ is $\Gamma$-invariant with positive measure. The action of $\Gamma$ on $X_{\Gamma}$ is ergodic for the measure $\tilde{\mu}$, hence $Z_{\epsilon}$ has full measure $\tilde{\mu}\left(Z_{\epsilon}\right)=1$.

Let $u$ be represented by the $\Gamma$-almost flat K-cocycle, $g^{a f}$, which determines a sequence of elements $\hat{g}_{i}=\pi^{*} g_{i} \in C_{\mathcal{F}_{\Gamma}}^{1}\left(\pi^{!} \mathbf{E}_{i}^{1}\right)$ with $\left\|\hat{g}_{i}\right\|_{(1)} \leq 1 / 4 i \kappa(\tilde{\mathcal{D}})$ and $\left[\hat{g}_{i}\right]=\left[\hat{g}_{0}\right] \in K_{a f}^{1}(V, \mathcal{F})$, where $\mathbf{E}_{i}^{1} \rightarrow M$ is a Hermitian flat bundle of dimension $N_{i}$. Fix an $i$ so that $1 / i<\epsilon$.

For $\mu$-almost every leaf $L$, the restriction $\pi^{!} \mathbf{E}_{i}^{1} \mid L$ is a Hermitian flat bundle with trivial holonomy, hence there is an Hermitian equivalence of flat bundles $U_{i}: \pi^{!} \mathbf{E}_{i}^{1} \cong L \times \mathbf{C}^{N_{i}}$. Let $\tilde{\mathcal{D}}^{1, i}$ denote the extension of $\tilde{\mathcal{D}}$ to sections of $\mathbf{E} \otimes \mathbf{E}_{i}^{1}$ as in the proof of Corollary 4.5. The existence of a leafwise unitary trivialization of the flat coefficient bundle then implies that $\sigma\left(\tilde{\mathcal{D}}_{L_{x}}\right)=\sigma\left(\tilde{\mathcal{D}}_{L_{x}}^{1, i}\right)$ for $\tilde{\mu}$-almost every $x$, and thus $\chi_{[\lambda, \lambda+\epsilon]}\left(\tilde{\mathcal{D}}_{L_{x}}^{1, i}\right)=0$ for $\tilde{\mu}$-almost every $x \in Z_{\epsilon}$. By formula $(25)$ it follows that the analytic $\mu$-index $T_{\mu}\left(\mathcal{I}\left(\tilde{\mathcal{D}}, g_{i}\right)\right)=0$.

On the other hand, the topological formula $(24)$ for $T_{\mu}\left(\mathcal{I}\left(\tilde{\mathcal{D}}, g_{i}\right)\right)$ depends only on the K-theory class of $\hat{g}_{i}$ and the principal symbol of $\tilde{\mathcal{D}}$, which is the lift of the symbol for $\mathcal{D}_{M}$. Hence, the topological pairing in (24) equals the pairing in (31) which does not vanish. This contradiction implies that that the set of leaves of $\mathcal{F}$ with gaps in their spectrum must have $\mu$-measure zero.

Our second result for coverings is an application of Theorem 4.3. We first formalize the expression appearing on the left-hand-side of $(27)$. Let $(V, \mathcal{F}, \mu)$ be a foliated measure space with an invariant transverse measure $\mu$. Fix a 
leafwise essentially self-adjoint, pseudo-differential operator $\mathcal{P} \in \Psi \mathcal{D} \mathcal{O}_{c}^{r}(\mathbf{E}, \mathcal{F})$ for some degree $r>0$. For example, let $\mathcal{P}=\tilde{\mathcal{D}}$ be a compact perturbation of a geometric operator. For $-\infty<\lambda_{0} \leq \lambda_{1}<\infty$, the family of leafwise spectral projections associated to $\mathcal{P}$ determines a class $\left[\chi_{\left[\lambda_{0}, \lambda_{1}\right]}(\mathcal{P})\right] \in K_{0}\left(W_{\mu}^{*}(V, \mathcal{F})\right)$, and we introduce the $\mu$-average spectral density function for $\mathcal{P}$ :

$$
\operatorname{Den}(V, \mathcal{F}, \mu)\left(\mathcal{P} ; \lambda_{0}, \lambda_{1}\right)=\operatorname{tr}_{\mu}\left(\left\{\chi_{\left[\lambda_{0}, \lambda_{1}\right]}\left(\mathcal{P}_{L}\right)\right\}\right)=T_{\mu}\left(\left[\chi_{\left[\lambda_{0}, \lambda_{1}\right]}(\mathcal{P})\right]\right)
$$

Consider the special case where $(V, \mathcal{F}, \mu)=\left(V_{\Gamma}, \mathcal{F}_{\Gamma}, \mu_{\Gamma}\right)$ and $\mathcal{P}$ is the leafwise $\Gamma$-periodic lift of a pseudo-differential operator $\mathcal{P}_{M}$ on $M$. We identify each leafwise operator $\mathcal{P}_{L}$ with the lift $\mathcal{P}_{\Gamma}$ of $\mathcal{P}_{M}$ to the covering $\tilde{M}_{\Gamma}$ with dense domain in the closure $\mathcal{H}_{\Gamma}$ of $C_{c}^{1}\left(\mathbf{E}_{\Gamma}\right)$. The trace $t r_{\mu_{\Gamma}}$ is then identified with the Atiyah's $\Gamma$-trace $\operatorname{tr}_{\Gamma}$ on $\mathcal{B}\left(\mathcal{H}_{\Gamma}\right)$, and we write

$$
\operatorname{Den}_{\Gamma}\left(\mathcal{P} ; \lambda_{0}, \lambda_{1}\right)=\operatorname{tr}_{\Gamma}\left(\chi_{\left[\lambda_{0}, \lambda_{1}\right]}\left(\mathcal{P}_{\Gamma}\right)\right)=T_{\mu_{\Gamma}}\left(\left[\chi_{\left[\lambda_{0}, \lambda_{1}\right]}(\mathcal{P})\right]\right)
$$

Let us note some elementary properties of the average density function (cf. $[39])$ :

PROPOSITION 5.3 $\operatorname{Den}(V, \mathcal{F}, \mu)\left(\mathcal{P} ; \lambda_{0}, \lambda_{1}\right)$ is monotone decreasing in $\lambda_{0}$ and monotone increasing in $\lambda_{1}$.

PROPOSITION 5.4 Let $(V, \mathcal{F})$ be a foliated measure space with a invariant transverse measure $\mu$, and let $\mathcal{P} \in \Psi \mathcal{D O}_{c}^{r}(\mathbf{E}, \mathcal{F})$ for $r>0$ be a leafwise essentially self-adjoint, pseudo-differential operator. If $\operatorname{Den}(V, \mathcal{F}, \mu)(\mathcal{P} ; \lambda, \lambda) \neq 0$, then there is a saturated subset $Z \subset V$ with positive $\mu$-measure, so that for each $L \subset Z$ the kernel of the operator $\mathcal{P}_{L}-\lambda$ has infinite multiplicity, spanned by smooth $L^{2}$-eigensections of $\mathbf{E}_{L} \rightarrow L$. That is, $\mathcal{P}_{L}$ has pure-point spectrum of energy $\lambda$ for all $L \subset Z$.

The works of Novikov \& Shubin [45, 44], Gromov \& Shubin [30] and Lott [42] study the asymptotic growth type about $\lambda=0$ of the function

$$
\epsilon \mapsto \operatorname{Den}_{\Gamma}\left(\Delta_{\Gamma} ; 0, \epsilon\right)
$$

where $\Delta_{\Gamma}$ is the lift to the covering $\tilde{M}_{\Gamma}$ of the Laplacian on forms on $M$. The asymptotic growth depends only on the germ of the function about $\epsilon=0$, and Gromov and Shubin prove that this germ is a topological invariant of the manifold.

The methods of this paper yield topological estimates for the germ of the function

$\operatorname{Den}_{\Gamma}\left(\Delta_{\Gamma} ; 0, \epsilon\right)$ about $\epsilon=0$ : the first is in terms of a new pseudo-norm on geometric operators; and then in the next section, we prove our most general spectral estimate for coverings in terms of the non-commutative isoperimetric function of section 2 .

Introduce the $\Gamma$ - $\epsilon$-almost flat pseudo-norm on the K-homology $K_{1}(M)$ of a compact manifold $M$ 


$$
\left\|\left[\mathcal{D}_{M}\right]\right\|_{\Gamma \epsilon}=\sup _{u \in K_{\Gamma a f}^{1}(M)}\left|\left\langle c h^{*}(u), c h_{*}\left(\left[\mathcal{D}_{M}\right]\right)\right\rangle\right| \cdot \mathcal{I}_{\Gamma, u}(\epsilon)
$$

The norm measures the "co-mass" of the K-homology cycle when paired with increasingly diffuse K-cocycles, so is similar to the Thurston norm [51] and Gromov norm [28] on homology. Proposition 2.9 and the topological invariance of the index class $c h_{*}\left(\left[\mathcal{D}_{M}\right]\right)$ (cf. [33]) imply

PROPOSITION 5.5 Let $M$ be a compact oriented manifold without boundary, and let $\Gamma$ be a group whose classifying space $B \Gamma$ is homotopic to a compact simplicial space. Then for a fixed surjection $\rho: \Lambda \rightarrow \Gamma$, the quasi-isometry class of the $\Gamma$-e-almost flat pseudo-norm on $K_{1}(M)$ is a topological invariant.

The next result gives a lower bound estimate for the spectral density function of a compact perturbation of a geometric operator $\mathcal{D}_{M}$ lifted to a normal covering $\tilde{M}_{\Gamma} \rightarrow M$, in terms of the $\Gamma$ - $\epsilon$-almost flat norm.

THEOREM 5.6 (Covering spectral estimate) Let $\tilde{\mathcal{D}}_{M}=\mathcal{D}_{M}+\mathcal{P}_{M}$ be a compact perturbation of a geometric operator on $C^{1}\left(\mathbf{E}_{M}\right)$ with $2 \epsilon\left(\mathcal{P}_{M}\right)$ less than the injectivity radius of $M$. Let $\tilde{M}_{\Gamma} \rightarrow M$ be a normal covering, with $\tilde{\mathcal{D}}_{\Gamma}$ the lift of $\tilde{\mathcal{D}}_{M}$ to $C_{c}^{1}\left(\mathbf{E}_{\Gamma}\right)$. Then for $\epsilon \leq\left(\lambda_{1}-\lambda_{0}\right) / 4 \kappa\left(\tilde{\mathcal{D}}_{M}\right)$

$$
\operatorname{Den}_{\Gamma}\left(\tilde{\mathcal{D}}_{\Gamma} ; \lambda_{0}, \lambda_{1}\right) \geq \frac{1}{4} \cdot\left\|\left[\mathcal{D}_{M}\right]\right\|_{\Gamma \epsilon}
$$

Proof: Lift the operator $\tilde{\mathcal{D}}_{M}$ to a leafwise operator $\tilde{\mathcal{D}}_{\Gamma}$ for $\left(V_{\Gamma}, \mathcal{F}_{\Gamma}, \mu_{\Gamma}\right)$. Let $\mathbf{E}_{M}^{1} \rightarrow M$ be an Hermitian flat bundle of dimension $N$ such that the holonomy homomorphism $\Lambda \rightarrow U(N)$ factors through the quotient group $\Gamma$. The restriction of the lift $\mathbf{E}^{1}=\pi^{!} \mathbf{E}_{M}^{1} \rightarrow V$ to a typical leaf of $\mathcal{F}_{\Gamma}$ is Hermitian trivial, and we can apply the method in the proof of Corollary 4.5 to obtain leafwise unitary equivalences as in (29).

Let $g \in C^{1}\left(U\left(\mathbf{E}_{M}^{1}\right)\right)$ with $\|g\|_{(1)}<\epsilon$ and lift $\hat{g} \in C_{\mathcal{F}_{\Gamma}}^{1}\left(\mathbf{E}^{1}\right)$. Set $K=\epsilon \kappa(\tilde{\mathcal{D}})$, then by Theorem 4.3

$$
\begin{aligned}
\left|\left\langle c h^{*}(u), c h_{*}\left(\left[\mathcal{D}_{M}\right]\right)\right\rangle\right| & =\left|\left\langle\psi^{-1}\left(c h^{*}\left(\sigma^{+}\left(\tilde{\mathcal{D}}_{M}^{1}, g\right)\right)\right) \cup \operatorname{Td}(T M),[M]\right\rangle\right| \\
& =\left|\left\langle\psi^{-1}\left(\operatorname{ch}^{*}\left(\sigma^{+}\left(\tilde{\mathcal{D}}_{\Gamma}^{1}, \hat{g}\right)\right)\right) \cup \operatorname{Td}\left(T \mathcal{F}_{\Gamma}\right),\left[C_{\mu_{\Gamma}}\right]\right\rangle\right| \\
& =\left|T_{\mu_{\Gamma}}(\mathcal{I}(\tilde{\mathcal{D}}, \hat{g}))\right| \\
& \leq 4 \cdot \operatorname{tr}_{\mu_{\Gamma}}\left(\left\{\chi_{[\lambda, \lambda+K]}\left(\tilde{\mathcal{D}}_{\Gamma}^{1}\right)\right\}\right) \\
& =4 N \cdot \operatorname{tr}_{\mu_{\Gamma}}\left(\left\{\chi_{[\lambda, \lambda+K]}\left(\tilde{\mathcal{D}}_{\Gamma}\right)\right\}\right)
\end{aligned}
$$

The last step uses the observation from the proof of Corollary 4.5 that the leafwise unitary maps $U_{L}$ induce a unitary in $W_{\mu}^{*}(V, \mathcal{F})$ conjugating the spectral 
projections of $\tilde{\mathcal{D}}_{\Gamma}^{1}$ with $N$ copies of those for the operator $\tilde{\mathcal{D}}_{\Gamma}$. Then from (36) and by Proposition 5.3, we have

$$
\left|\left\langle c h^{*}(u), c h_{*}\left(\left[\mathcal{D}_{M}\right]\right)\right\rangle\right| \leq 4 N \cdot \operatorname{tr}_{\mu_{\Gamma}}\left(\left\{\chi_{\left[\lambda_{0}, \lambda_{1}\right]}\left(\tilde{\mathcal{D}}_{\Gamma}\right)\right\}\right)
$$

from which (35) is deduced.

\section{Application to random perturbations}

The results of the last section addressed the spectral properties of a $\Gamma$ periodic geometric operator on a normal covering $\tilde{M}_{\Gamma}$. In this section, we show how the results of section 4 can be applied to geometric operators on $\tilde{M}_{\Gamma}$ which are obtained from random perturbations of the lift of the Riemannian metric to $T \tilde{M}_{\Gamma}$. This case has relevance to problems in solid state physics (cf. [5]; Chapter $4,[16]$ ), where spectral density results for random geometric operators imply conductivity estimates for electron models in the presence of a force field.

Fix a quotient group $\rho: \Lambda \rightarrow \Gamma$ and a Riemannian metric $h_{M}$ on $T M$, with lift $h_{\Gamma}$ to the covering $T \tilde{M}_{\Gamma}$. The idea of a "random perturbation" $\tilde{h}$ of a tensor field on $\tilde{M}_{\Gamma}$ such as $h_{\Gamma}$ is that it should be close to the original metric in some sense, and the set of translates $\{\gamma \cdot \tilde{h} \mid \gamma \in \Gamma\}$ should be pre-compact in a suitable topology. Moreover, we demand that the "closure" of $\{\gamma \cdot \tilde{h} \mid \gamma \in \Gamma\}$ be a Borel measure space $\Omega$ - the space of nearby random perturbations - endowed with a $\Gamma$-invariant measure. These ideas are discussed further in (section $5,[39]$ ) for random perturbations of unitary multipliers on $\tilde{M}_{\Gamma}$. We use the following definition:

DEFINITION 6.1 $A$ random perturbation of $h_{\Gamma}$ consists of the data $\left(\Omega, \tilde{\mu}, \varphi, h_{\mathcal{F}_{\varphi}}\right)$, where

- $(\Omega, \tilde{\mu})$ is a Borel measure space of total mass 1

- $\varphi: \Gamma \times \Omega \rightarrow \Omega$ is an ergodic action preserving the measure $\tilde{\mu}$

- $h_{\mathcal{F}_{\varphi}}$ is a measurable family of $C^{2}$-Riemannian metrics on the tangent spaces $T \mathcal{F}_{\varphi}$ of the foliation on $V_{\varphi}$ constructed from the suspension of $\varphi$

- There exists a constant $K>0$ so that for each leaf $L$, the restriction $h_{L}$ of $h_{\mathcal{F}_{\varphi}}$ to $L$ is within distance $K$ of the lifted metric $h_{\Gamma}$, for the uniform $C^{2}$-topology on tensors.

The metrics are required to be at least $C^{2}$ in the definition, in order to guarantee that the corresponding Dirac operators have at least $C^{1}$-coefficients, hence are essentially self-adjoint. 
THEOREM 6.2 Let $\mathcal{D}_{M}$ be a geometric operator associated with the Riemannian metric $h_{M}$ on TM and a Hermitian coefficient bundle $\mathbf{E}_{M}^{0} \rightarrow M$, and $u \in K_{\Gamma a f}^{1}(M) \subset K^{1}(M)$ such that $\left\langle\operatorname{ch}^{*}(u), \operatorname{ch}_{*}\left(\left[\mathcal{D}_{M}\right]\right)\right\rangle \neq 0$ and

- $\left(\Omega, \tilde{\mu}, \varphi, h_{\mathcal{F}_{\varphi}}\right)$ a random perturbation of $h_{\Gamma}$

- $\mathcal{D}$ the associated leafwise geometric operator on $\mathcal{F}_{\varphi}$ with coefficients in $\mathbf{E}=\pi^{!} \mathbf{E}_{M}$

- $\tilde{\mathcal{D}}=\mathcal{D}+\mathcal{P}$ a compact perturbation of $\mathcal{D}$ with $2 \epsilon(\mathcal{P})$ less than the injectivity radius of $M$,

the spectrum $\sigma\left(\tilde{\mathcal{D}}_{L}\right)=\mathbf{R}$ for $\mu$-almost every leaf $L \subset V_{\varphi}$. Moreover, there is a lower bound for the average spectral density function, for all $\lambda \in \mathbf{R}$ and $\epsilon>0$

$$
\operatorname{Den}\left(V_{\varphi}, \mathcal{F}_{\varphi}, \mu\right)(\tilde{\mathcal{D}} ; \lambda, \lambda+\epsilon) \geq \frac{1}{4}\left|\left\langle c h^{*}(u), \operatorname{ch}_{*}\left(\left[\mathcal{D}_{M}\right]\right)\right\rangle\right| \cdot \mathcal{I}_{\Gamma, u}(\epsilon / 4 \kappa(\tilde{\mathcal{D}}))
$$

Proof: The coefficients of the leafwise foliation Dirac operator $\mathcal{D}_{L}$ depend continuously on metric $h$ on $T \mathcal{F}_{\varphi}$, for the $C^{1}$-topology on tensors, so the coefficients of a random perturbation $\mathcal{D}$ vary measurably within a bounded distance of those for the $\Gamma$-periodic operator $\mathcal{D}_{\Gamma}$. It follows that the "random geometric operator" $\mathcal{D}$ is leafwise homotopic in the transversally measurable category to the $\Gamma$-periodic operator $\mathcal{D}_{\Gamma}$. Let $u$ be as in the theorem, represented by a $\Gamma$-almost flat K-cocycle $g^{a f}$ which determines lifts $\hat{g}_{i} \in C_{\mathcal{F}_{\varphi}}^{1}\left(\mathbf{E}^{1}\right)$ to $\left(V_{\varphi}, \mathcal{F}_{\varphi}\right)$ with $\left\|\hat{g}_{i}\right\|_{(1)}<1 / i$. Recall that $\left[\hat{g}_{i}\right]=\left[\hat{g}_{1}\right] \in K_{a f}^{1}\left(V_{\varphi}, \mathcal{F}_{\varphi}\right)$ is constant in $i$, so that the topological Toeplitz index class $c h^{*}\left(\sigma^{+}\left(\mathcal{D}^{1}, \hat{g}_{i}\right)\right)$ is also independent of $i$, and cohomologous in the leafwise deRham complex to that for the periodic lift $\mathcal{D}_{\Gamma}$. Thus, the cohomology index pairing

$$
(-1)^{m}\left\langle\psi^{-1}\left(\operatorname{ch}^{*}\left(\sigma^{+}\left(\mathcal{D}^{1}, \hat{g}_{i}\right)\right)\right) \cup \operatorname{Td}\left(\mathcal{F}_{\varphi}\right),\left[C_{\mu}\right]\right\rangle=\left\langle c h^{*}\left(\left[g^{a f}\right]\right), c h_{*}\left(\left[\mathcal{D}_{M}\right]\right)\right\rangle \neq 0
$$

Now apply Corollary 4.4 to obtain that $\sigma\left(\tilde{\mathcal{D}}_{L}\right)=\mathbf{R}$ for almost every leaf $L$.

The proof of the spectral density estimate (38) follows from Corollary 4.5, where we note that $\mathcal{I}_{\mathcal{F} \hat{g}_{1}}(\epsilon) \geq \mathcal{I}_{\Gamma g_{1}}(\epsilon)$.

Theorem 1.1 of the Introduction is deduced from Theorem 6.2 by taking $\Omega=X_{\Gamma}$ and letting $h_{\mathcal{F}_{\varphi}}=h_{\Gamma}$ be the periodic lift of a metric from $M$. The estimate (1) of Theorem 1.1 for $\tilde{\mathcal{D}}=\tilde{\mathcal{D}}_{\Gamma}$ is a consequence of (38).

\section{$7 \quad$ Examples}

EXAMPLE 1 (Periodic Operators on $\mathbf{R}^{m}$ ) The universal covering of the m-torus $\mathbf{T}^{m}$ is $\mathbf{R}^{m}$ with the fundamental group $\Lambda$ of $\mathbf{T}^{m}$ identified with the covering group $\mathbf{Z}^{m}$ acting by translations. A Riemannian metric on $\mathbf{T}^{m}$ lifts to a $\mathbf{Z}^{m}$-periodic Riemannian metric on $\mathbf{R}^{m}$, and an Hermitian vector bundle $\mathbf{E}_{\mathbf{T}^{m}}^{0} \rightarrow \mathbf{T}^{m}$ lifts to a $\mathbf{Z}^{m}$-periodic coefficient system $\mathbf{E}^{0} \rightarrow \mathbf{R}^{m}$. 
A more general construction is to introduce a random perturbation (cf. Chapter 9 of [21]) of the $\mathbf{Z}^{m}$-periodic operator $\mathcal{D}_{\mathbf{Z}^{m}}$, in the form of the data $\left(\Omega, \tilde{\mu}, \varphi, h_{\mathcal{F}_{\varphi}}\right)$. Each $x \in \Omega$ determines a leaf $L_{x}$ and by restriction a geometric operator $\tilde{\mathcal{D}}_{x}$ on $L_{x} \cong \mathbf{R}^{m}$, which is a compact perturbation of $\tilde{\mathcal{D}}_{x}$ acting on the sections over the leaf $L_{x}$. The action of $\vec{v} \in \mathbf{Z}^{m}$ on $\mathbf{R}^{m}$ translates $\tilde{\mathcal{D}}_{x}$ to the operator $\tilde{\mathcal{D}}_{\vec{v} \cdot x}$. Restricting $\tilde{\mathcal{D}}$ to the leaves of $\mathcal{F}_{\varphi}$ through the orbit of $x \in \Omega$ thus yields the set of translates $\left\{\tilde{\mathcal{D}}_{\vec{v} \cdot x} \mid \vec{v} \in \mathbf{Z}^{m}\right\}$, which is a random sequence of operators. An almost periodic perturbation is a random perturbation $\tilde{\mathcal{D}}$ where $\Omega$ is a compact connected, locally connected abelian group, the action of $\mathbf{Z}^{m}$ is transitive on $\Omega$ and the operator $\tilde{\mathcal{D}}$ has continuous coefficients. One observes that the set of translates $\left\{\tilde{\mathcal{D}}_{\vec{v} \cdot x} \mid \vec{v} \in \mathbf{Z}^{m}\right\}$ is precompact in the uniform topology, so this recovers the usual notion of almost periodic operators.

A typical example is to give $\mathbf{T}^{m}$ the standard flat metric, and introduce an almost-periodic "force field" on $\mathbf{R}^{m}$. That is, we take an almost-periodic Hermitian coefficient system $\mathbf{E}^{0} \rightarrow \mathbf{R}^{n}$ with an Hermitian connection $\nabla^{\mathbf{E}^{0}}$. Then the lifted flat Dirac operator couples to the bundle $\mathbf{E}^{0}$ to yield a geometric operator $\mathcal{D}_{\mathbf{Z}^{m}}=\mathcal{D}_{\mathbf{Z}^{m}} \otimes \nabla^{\mathbf{E}^{0}}$ on $\mathbf{R}^{m}$ which is the quantum almost-periodic generator for a particle moving in the "force field" $\nabla^{\mathbf{E}^{0}}$.

THEOREM 7.1 Let $h_{\mathbf{Z}^{m}}$ be a $\mathbf{Z}^{m}$-periodic Riemannian metric on $\mathbf{R}^{m}$ for $m$ odd, and $\mathcal{D}_{\mathbf{T}^{m}}$ a geometric operator on $\mathbf{T}^{m}$.

1. $\sigma\left(\tilde{\mathcal{D}}_{\mathbf{Z}^{m}}\right)=\mathbf{R}$ for $\tilde{\mathcal{D}}_{\mathbf{Z}^{m}}$ the $\mathbf{Z}^{m}$-periodic lift to $\mathbf{R}^{m}$ of a compact perturbation of $\mathcal{D}_{\mathbf{T}^{m}}$.

2. $\sigma\left(\tilde{\mathcal{D}}_{x}\right)=\mathbf{R}$ for almost every $x \in \Omega$, where $\tilde{\mathcal{D}}$ is a compact perturbation of the lifted geometric operator $\tilde{\mathcal{D}}_{\mathcal{F}}$ associated to a random perturbation $\left(\Omega, \tilde{\mu}, \varphi, h_{\mathcal{F}_{\varphi}}\right)$ of the periodic metric $h_{\mathbf{T}^{m}}$.

Proof: The conclusions will follow from Theorems 5.1 and 6.2 , if we show the existence of a class $u \in K_{a f}^{1}\left(\mathbf{T}^{m}\right)$ so that $\left\langle c h^{*}(u), c h_{*}\left(\left[\mathcal{D}_{\mathbf{T}^{m}}\right]\right)\right\rangle \neq 0$. First note that for an arbitrary compact Spin manifold $M$, the odd index pairing Ind : $K^{1}(M) \otimes K_{1}(M) \rightarrow \mathbf{Z}$ is non-singular with the formula (Theorem 4, [4])

$$
\begin{aligned}
& \left\langle c h^{*}(u), \operatorname{ch}_{*}\left(\left[\mathcal{D} \otimes \nabla^{\left.\left.\left.\mathbf{E}_{M}^{0}\right]\right)\right\rangle=}\right.\right.\right. \\
& \quad\left\langle c h^{*}(u) \cup \operatorname{ch}^{*}\left(\left[\mathbf{E}_{M}^{0}\right]\right) \cup \operatorname{ch}^{*}\left(\sigma^{+}([\mathcal{D}])\right) \cup T d(T M),[M]\right\rangle
\end{aligned}
$$

Thus, given any geometric operator $\mathcal{D}_{\mathbf{T}^{m}}$ there is $u \in K^{1}\left(\mathbf{T}^{m}\right)$ so that $\left\langle\operatorname{ch}^{*}(u), \operatorname{ch}_{*}\left(\left[\mathcal{D}_{\mathbf{T}^{m}}\right]\right)\right\rangle \neq 0$. Represent $u$ by a $C^{1}$-map $g_{0}: \mathbf{T}^{m} \rightarrow U((m+1) / 2)$.

We follow the re-scaling method of of Gromov and Lawson [29]: For each integer $i>0$ let $\pi_{i}: \tilde{M}_{i} \rightarrow \mathbf{T}^{m}$ denote the covering corresponding to the subgroup $\Lambda_{i}=i \cdot \mathbf{Z}^{m} \subset \mathbf{Z}^{m}$ with index $\left[\Lambda: \Lambda_{i}\right]=i^{m}$. Let $\Phi_{i}: \tilde{M}_{i} \cong \mathbf{T}^{m}$ be the canonical diffeomorphism which decreases distances by the factor $1 / i$ and define a unitary $g_{i}=g_{0} \circ \Phi_{i}: \tilde{M}_{i} \rightarrow U$. Thus, each map $g_{i}$ is topologically the same as the map $g_{0}$ but is considered as a map on the covering $\tilde{M}_{i}$ which is a 
metric re-scaling of the base torus. The sequence $g^{p f}=\left\{\left(g_{i}, \Lambda_{i}, N_{i}\right) \mid 0 \leq i\right\}$ for $N_{i}=(m+1) / 2$ clearly satisfies the conditions of Definition 2.12.

Given a $C^{1}$-map $g: M \rightarrow U(p)$, set

$$
\left\|\left[g_{0}\right]\right\|_{(1)}=\inf \left\{\|g\|_{(1)} \mid g: M \rightarrow U(p) \text { and } g \sim g_{0}\right\}
$$

Combine the proof of Theorem 7.1 with that of Proposition 2.13 to obtain:

PROPOSITION 7.2 Let $0 \neq u \in K^{1}\left(\mathbf{T}^{m}\right)$ be represented by $g_{0}: \mathbf{T}^{m} \rightarrow$ $U((m+1) / 2)$.

$$
D_{\mathbf{Z}^{m}, u}\left(\left\|\left[g_{0}\right]\right\|_{(1)} / \ell\right) \leq \frac{(m+1) \cdot \ell^{m}}{2}
$$

and hence $\mathcal{I}_{\mathbf{Z}^{m}, u}(\epsilon) \sim \epsilon^{m}$ for $\epsilon$ small.

COROLLARY 7.3 Let $h_{\mathbf{Z}^{m}}$ be a $\mathbf{Z}^{m}$-periodic Riemannian metric on $\mathbf{R}^{m}$ for $m$ odd. Let $\mathcal{D}_{\mathbf{T}^{m}}$ be a geometric operator on $\mathbf{T}^{m}$, and $g_{0}: \mathbf{T}^{m} \rightarrow U((m+1) / 2)$ so that $\left\langle\operatorname{ch}^{*}\left(\left[g_{0}\right]\right), \operatorname{ch}_{*}\left(\left[\mathcal{D}_{\mathbf{T}^{m}}\right]\right)\right\rangle \neq 0$. Let $\tilde{\mathcal{D}}=\mathcal{D}+\mathcal{P}$ be a compact perturbation of the geometric operator on $\mathcal{F}_{\varphi}$ associated to a random perturbation $h_{\mathcal{F}_{\varphi}}$ of the periodic metric $h_{\mathbf{Z}^{m}}$. Then for $\lambda_{1}-\lambda_{0}>4\left\|\left[g_{0}\right]\right\|_{(1)} \kappa(\tilde{\mathcal{D}}) / \ell$,

$$
\operatorname{Den}\left(V_{\varphi}, \mathcal{F}_{\varphi}, \mu\right)\left(\tilde{\mathcal{D}} ; \lambda_{0}, \lambda_{1}\right) \geq \frac{1}{2(m+1) \ell^{m}}
$$

REMARK 7.4 The motivation for calling $\mathcal{I}_{\Gamma, u}(\epsilon)$ an "isoperimetric function" appears in the above derivation of the estimate (40). Recall the usual isoperimetric constant for a complete Riemannian manifold $X$ (cf. Theorem 1, [53]):

$$
h(X)=\inf _{U \subset X} \inf _{f \in C_{c}^{1}(U)} \frac{\int_{U}\|\nabla f\| d v o l}{\int_{U}|f| d v o l}
$$

For a typical test function $f$ which satisfies $|f| \leq 1$, the isoperimetric constant is dominated by the ratio of the supremum of $\|\nabla f\|$ on $U$ to the mass of $U$. Observe that for a class $u \in K_{\Gamma p f}^{1}(M)$, the function $\mathcal{I}_{\Gamma, u}(\epsilon)$ is dominated by the ratio of the supremum of $\|\nabla g\|$, for $g: \tilde{M}_{i} \rightarrow U(\infty)$ in the class of $u$, to the number $\left|\Gamma_{i}\right|$ which is proportional to the mass of $\tilde{M}_{i}$.

EXAMPLE 2 (Compactly enlargeable manifolds) The above example $M=\mathbf{T}^{m}$ is a special case of a general class of manifolds for which one can derive an estimate for $\mathcal{I}_{\Lambda, u}(\epsilon)$. Recall the definition of an a compactly enlargeable manifold due to Gromov and Lawson ([29]; see also page 302, [41]). A Riemannian manifold is enlargeable of dimension $m$ if for every $\epsilon>0$, there exists a covering (possibly infinite) $\tilde{M}_{\epsilon} \rightarrow M$ and a degree one map $f_{\epsilon}: \tilde{M}_{\epsilon} \rightarrow S^{m}$ which is constant at infinity and has $\left\|\nabla f_{\epsilon}\right\|<\epsilon$. The manifold $M$ is compactly enlargeable if for each $\epsilon>0$, there exists a finite covering $\tilde{M}_{\epsilon}$ with these properties. There are many examples of compactly enlargeable manifolds: 
THEOREM 7.5 (Theorems 5.3, 5.4, [41]) The following are compactly enlargeable:

1. A compact Riemannian manifold which admits a globally expanding selfmap.

2. A compact arithmetic manifold with constant non-positive sectional curvatures.

3. The product of compactly enlargeable manifolds.

4. The connected sum of any compact manifold with a compactly enlargeable manifold.

5. Any manifold which admits a map of non-zero degree onto an enlargeable manifold.

Recall that a map $f$ from a metric space $\left(X, d_{X}\right)$ to a metric space $\left(Y, d_{Y}\right)$ is called globally expanding if for any two points $x_{1}, x_{2} \in X$ with $x_{1} \neq x_{2}$, one has $d_{Y}\left(f\left(x_{1}\right), f\left(x_{2}\right)\right)>d_{X}\left(x_{1}, x_{2}\right)$. John Franks proved that the fundamental group $\Lambda=\pi_{1}\left(M, x_{0}\right)$ of a compact Riemannian manifold $M$ which admits a globally expanding self-map has polynomial growth, hence by the celebrated theorem of Gromov, $\Lambda$ must contain a nilpotent subgroup of finite index. Thus, by Shub's criteria the map $f$ is topologically conjugate to an expanding infranil-endomorphism of $M$. See the Introduction and section 1 of the paper of Gromov, [27], for a discussion and references concerning globally expanding self-maps.

The covering degree function of a compactly enlargeable Riemannian manifold $M$ is defined for all $\epsilon>0$ :

$$
\begin{array}{r}
C D_{M}(\epsilon)=\inf \left\{\left[\Lambda: \Lambda_{i}\right] \mid \Lambda_{i}=\pi_{1}\left(\tilde{M}_{i}\right)\right. \text { and there exists a degree one map } \\
\left.\qquad f_{\epsilon}: \tilde{M}_{i} \rightarrow S^{m} \text { with }\left\|\nabla f_{\epsilon}\right\|<\epsilon\right\} .
\end{array}
$$

The proof of Proposition 2.13 yields the estimate:

LEMMA 7.6 Let $M$ be a compactly enlargeable, odd dimensional Riemannian manifold with fundamental group $\Lambda$, and $u=[\iota \circ f] \in K^{1}(M)$ the K-theory class of $\iota: S^{m} \rightarrow U((m+1) / 2)$ composed with a degree-one map $f: M \rightarrow S^{m}$. Then there exists a constant $C(M)>0$ so that

$$
D_{\Lambda, u}(\epsilon) \leq C(M) \cdot C D_{M}(\epsilon)
$$

In particular, this implies that the reciprocal function $\mathcal{I}_{\Lambda, u}(\epsilon)>0$ when $\epsilon>0$.

COROLLARY 7.7 Let $M$ be a compactly enlargeable Riemannian manifold of odd dimension $m, f: M \rightarrow S^{m}$ a degree-one map, and $\mathcal{D}_{M}$ a geometric operator on $M$ so that the push-forward class in K-homology $f_{*}\left[\mathcal{D}_{M}\right] \in K_{1}\left(S^{m}\right)$ is nonzero. Let $\tilde{\mathcal{D}}_{M}=\mathcal{D}_{M}+\mathcal{P}_{M}$ be a compact perturbation of $\mathcal{D}_{M}$, with $2 \epsilon\left(\mathcal{P}_{M}\right)$ less than the injectivity radius of $M$. 
1. $\sigma\left(\tilde{\mathcal{D}}_{\Lambda}\right)=\mathbf{R}$ for $\tilde{\mathcal{D}}_{\Lambda}$ the lift of $\tilde{\mathcal{D}}_{M}$ to a periodic operator on the universal covering of $M$,

2. for $u=\left[g_{0}\right]$ where $g_{0}=\iota \circ f: M \rightarrow U((m+1) / 2)$ there is a lower bound estimate on the $\Lambda$-spectral density function

$$
\operatorname{tr}_{\Lambda}\left(\chi_{[\lambda, \lambda+\epsilon)}\left(\tilde{\mathcal{D}}_{\Lambda}\right)\right) \geq \frac{\left\langle c h^{*}(u), c h_{*}\left(\left[\mathcal{D}_{M}\right]\right)\right\rangle}{2(m+1) C(M) \cdot C D_{M}\left(\epsilon / 4 \kappa\left(\tilde{\mathcal{D}}_{\Lambda}\right)\right)}>0
$$

REMARK 7.8 Theorem 6.9 of Roe [49] implies that $\sigma(\mathcal{D})=\mathbf{R}$ for a random geometric perturbation $\mathcal{D}$ of $\tilde{\mathcal{D}}_{\Lambda}$, which implies the conclusion of Corollary 7.7.1. It is an appropriate point to compare the foliation index method of this paper and the exotic index method of Roe. The homology Chern character of a geometric operator $\mathcal{D}_{\Gamma}$ on $\tilde{M}_{\Gamma}$ lies in the homology theory of locally-finite, but not necessarily compactly supported chains (cf. comments in section 4, [24]). Both approaches build upon this basic homological data, and the strategy is to formulate a cohomology theory that pairs with such chains, then relate the values of the pairing to the spectrum. In coarse cohomology, the pairing factors through the complex of cochains with compact supports on $\tilde{M}_{\Gamma}$. Thus, the theory detects the non-triviality of index via an essentially local method. However, one of the main points of coarse cohomology theory is that there is a subcomplex of the complex of compactly supported cochains whose cohomology groups "come from infinity"; or more precisely, from the Higson Corona of $\tilde{M}_{\Gamma}$ (cf. section $5,[49])$. The compactly supported classes which arise from the Higson Corona pair trivially with a K-homology class that arises from a geometric operator with a gap in its spectrum, and this is the source of the "no-gap" theorems proved using coarse cohomology theory.

The method of this paper pairs homology with infinite supports with renormalized bounded cochains on $\tilde{M}_{\Gamma}$. This requires that the terms in the pairing be "renormalizable", which in practice requires that the data on $\tilde{M}_{\Gamma}$ be the restriction to a leaf $L \cong \tilde{M}_{\Gamma}$ of global data defined on a foliated manifold $\left(V_{\varphi}, \mathcal{F}_{\varphi}, \mu\right)$ with an invariant transverse measure (cf. discussion of section 5, [34]). The "renormalization" aspect of the foliation index theorem pairing implies that it also detects classes "from infinity". On the analytical level, renormalization amounts to forming a sequence of Toeplitz compression operators using multipliers whose gradients tend to zero, and hence the pairing localizes to a given region of the spectrum. The existence of a compact setting for the analysis, the foliated total space $V_{\varphi}$, conveys an additional advantage: the renormalization procedure produces an explicit measure of the renormalized spectral density, so that the method yields more than simply the assertion that the spectrum is all of $\mathbf{R}$. 
EXAMPLE 3 (Partially enlargeable manifolds) The next family of examples consist of manifolds which map to the examples in Example 2. We say that $M$ is partially compactly enlargeable if there exists a continuous map $q: M \rightarrow B$ to a compactly enlargeable manifold $B$ inducing a surjection on fundamental groups.

THEOREM 7.9 Let $M$ be partially compactly enlargeable, with $B$ a compactly enlargeable manifold with fundamental group $\Gamma=\pi_{1}\left(B, b_{0}\right)$, and $q: M \rightarrow B$ a continuous map inducing a surjection $q_{\#}: \Lambda \rightarrow \Gamma$ on fundamental groups. Denote the kernel of $q_{\#}$ by $\Lambda(q) \subset \Lambda$. Suppose that $\mathcal{D}_{M}$ is a geometric operator on $M$ with the push-forward class in K-homology $\left(g_{0} \circ q\right)_{*}\left[\mathcal{D}_{M}\right] \in K_{1}\left(S^{m}\right)$ nonzero for a degree-one map $g_{0}: B \rightarrow S^{m}$. Then for every normal covering $\tilde{M}_{\Gamma^{\prime}} \rightarrow$ $M$ with the kernel of $\Lambda \rightarrow \Gamma^{\prime}$ contained in $\Lambda(q)$ :

1. $\sigma\left(\tilde{\mathcal{D}}_{\Gamma^{\prime}}\right)=\mathbf{R}$ for $\tilde{\mathcal{D}}_{\Gamma^{\prime}}$ the lift to $\tilde{M}_{\Gamma^{\prime}}$ of a compact perturbation of $\mathcal{D}_{M}$,

$$
\operatorname{tr}_{\Gamma^{\prime}}\left(\left\{\chi_{[\lambda, \lambda+\epsilon)}\left(\tilde{\mathcal{D}}_{\Gamma^{\prime}}\right)\right\}\right) \geq \frac{\left|\left\langle c h^{*}\left(\left[\iota \circ g_{0} \circ q\right]\right), c h_{*}\left(\left[\mathcal{D}_{M}\right]\right)\right\rangle\right|}{4 N_{u} \cdot C D_{M}\left(\epsilon / 4 \kappa\left(\tilde{\mathcal{D}}_{\Gamma^{\prime}}\right)\right)}
$$

2. $\sigma\left(\tilde{\mathcal{D}}_{L}\right)=\mathbf{R}$ for almost every $L \subset V_{\varphi}$, where $\tilde{\mathcal{D}}$ is a compact perturbation of the geometric operator associated to a random perturbation $h_{\mathcal{F}_{\varphi}}$ of a periodic metric $h_{\Gamma^{\prime}}$ on $\tilde{M}_{\Gamma^{\prime}}$.

Let us point out a simple application of Theorem 7.9.

COROLLARY 7.10 Let $M$ be a compact manifold admitting a continuous map $q: M \rightarrow \mathbf{T}^{m}$ which induces a surjection $q_{\#}: \Lambda \rightarrow \mathbf{Z}^{m}$ on fundamental groups, with kernel $\Lambda(q) \subset \Lambda$. Suppose that $\mathcal{D}_{M}$ is a geometric operator on $M$ so that for the top dimensional class $u \in K^{1}\left(\mathbf{T}^{m}\right)$, represented by $\iota \circ g_{0}: \mathbf{T}^{m} \rightarrow$ $U(N)$,

$$
\left\langle\operatorname{ch}^{*}\left(q^{!} u\right), \operatorname{ch}_{*}\left(\left[\mathcal{D}_{M}\right]\right)\right\rangle \neq 0 .
$$

Then for every normal covering $\tilde{M}_{\Gamma^{\prime}} \rightarrow M$ with the kernel of $\Lambda \rightarrow \Gamma^{\prime}$ contained in $\Lambda(q)$, there is a uniform estimate on the $\Gamma^{\prime}$-spectral density function of an operator $\tilde{\mathcal{D}}_{\Gamma^{\prime}}$ which is the lift to $\tilde{M}_{\Gamma^{\prime}}$ of a compact perturbation of the geometric operator $\mathcal{D}_{M}$, for $\ell>0$ and $\epsilon(\ell)=4 \kappa\left(\tilde{\mathcal{D}}_{\Gamma^{\prime}}\right) / \ell$

$$
\operatorname{tr}_{\Gamma^{\prime}}\left(\left\{\chi_{[\lambda, \lambda+\epsilon(\ell))}\left(\tilde{\mathcal{D}}_{\Gamma^{\prime}}\right)\right\}\right) \geq \frac{\left|\left\langle c h^{*}\left(q^{!} u\right), c h_{*}\left(\left[\mathcal{D}_{M}\right]\right)\right\rangle\right|}{4 N \cdot \ell^{m}}
$$

It is not known whether there exists a normal covering $\tilde{M}_{\Gamma^{\prime}}$ with no coarse fundamental class. The existence of such a covering would provide an example of a geometric operator on an open manifold where the renormalization method of this paper implies that $\sigma\left(\tilde{\mathcal{D}}_{\Gamma^{\prime}}\right)=\mathbf{R}$, but the coarse cohomology method of Roe does not apply. 
EXAMPLE 4 (Asymptotic estimates) The spectral density estimate (35) involves the Chern character of the defining data for the elliptic problem. This allows one to construct estimates on the spectral density function which grow with the "degree" of the data, in a way reminiscent of the various Demailley estimates for holomorphic bundles $[22,50]$.

Let $\Sigma$ denote a compact Riemann surface of genus $\geq 2$, and let $\kappa^{\otimes p} \rightarrow \Sigma$ denote the $p^{t h}$-power of the canonical bundle. We let $M=\Sigma \times S^{1}$ be the product of the surface with a circle, and choose a Riemannian metric $h_{M}$ on $T M$. The fundamental group $\Lambda=\pi_{i}(\Sigma) \times \mathbf{Z}$, so there is a natural surjection onto $\mathbf{Z}$ with kernel $\pi_{1}(\Sigma)$. Apply Corollary 7.10 to obtain

THEOREM 7.11 Let $\partial_{M}^{\otimes p}$ be the geometric operator obtained from the Dirac operator for $h_{M}$ with coefficients in the lifted bundle $\kappa^{\otimes}$. Let $\tilde{M}_{\Gamma}$ be a normal covering associated to a homomorphism $\Lambda \rightarrow \Gamma$ whose kernel is contained in $\pi_{1}(\Sigma)$.

1. $\sigma\left(\tilde{\mathcal{D}}_{\Gamma}^{\otimes p}\right)=\mathbf{R}$ for each operator $\tilde{\mathcal{D}}^{\otimes p}$ which is the lift to $\tilde{M}_{\Gamma}$ of a compact perturbation of the operator $\partial_{M}^{\otimes p}$.

2. $\sigma\left(\tilde{\mathcal{D}}_{L}^{\otimes p}\right)=\mathbf{R}$ for almost every $L \subset V_{\varphi}$ when $\tilde{\mathcal{D}}^{\otimes p}$ is a compact perturbation of the geometric operator associated to a random perturbation $h_{\mathcal{F}_{\varphi}}$ of the periodic metric $h_{\Gamma^{\prime}}$ on $\tilde{M}_{\Gamma}$.

3. The spectral density function of a random perturbation $\tilde{\mathcal{D}}^{\otimes p}$ of $\partial_{\Gamma}^{\otimes p}$ satisfies

$$
\operatorname{Den}\left(V_{\varphi}, \mathcal{F}_{\varphi}, \mu\right)\left(\tilde{\mathcal{D}}^{\otimes p} ; \lambda_{0}, \lambda_{1}\right) \geq \frac{p}{4 \ell}
$$

whenever $\lambda_{1}-\lambda_{0}>4 \kappa\left(\tilde{\mathcal{D}}^{\otimes p}\right) / \ell$.

\section{References}

[1] M. F. Atiyah. Elliptic operators, discrete groups and von Neumann algebras. pages 43-72, 1976. Astèrisque 32-33.

[2] M. F. Atiyah. Eigenvalues of the Dirac operator. In Proceedings of the 25 th Mathematics Arbeitstagung, Bonn 1984, pages 251-260, 1985. Lecture Notes in Math. vol. 1111.

[3] P. Baum and R. G. Douglas. K-homology and index theory. In Operator algebras and applications, pages 117-173, 1982. Proc. Symp. Pure Math. vol. 38, Part 1.

[4] P. Baum and R. G. Douglas. Toeplitz operators and Poincaré duality. In Proc, Toeplitz Memorial Conf., Tel Aviv 1981, pages 137-166, Basel, 1982. Birkhäuser.

[5] J. Bellisard. K-theory of $C^{*}$-algebras in solid-state physics, statistical mechanics and field theory, mathematical aspects. pages 99-156. Springer-Verlag, 1986. Lect. Notes in Phys. vol. 257.

[6] M. Breuer. Fredholm theories in von Neumann algebras I. Math. Annalen, 178:243-254, 1968 
[7] M. Breuer. Fredholm theories in von Neumann algebras II. Math. Annalen, 180:313-325, 1969

[8] R. Brooks. The fundamental group and the spectrum of the Laplacian. Comment. Math. Helv., 56:581-598, 1981.

[9] R. Brooks. The spectral geometry of foliations. American Jour. Math., pages 1001-1012, 1983

[10] U. Bunke. On the spectral flow of families of Dirac operators with constant symbol. preprint - December,1991, Max-Planck-Institut, MPI/91-86.

[11] C. Camcho and Neto. Geometric Theory of Foliations. Progress in Math. Birkhäuser, Boston, Basel and Stuttgart, 1985.

[12] A. Connes. The von Neumann algebra of a foliation. pages 145-151, New York and Berlin, 1978. Springer-Verlag. Lect. Notes in Physics vol. 80.

[13] A. Connes. Sur la théorie non-commutative de l'integration, Algèbres d'opérateurs. pages 19-143. Springer-Verlag, 1979. Lect. Notes in Math. vol. 725 .

[14] A. Connes. A survey of foliations and operator algebras. In Operator Algebras and Applications, pages 521-628. Amer. Math. Soc., 1982. Proc. Symp. Pure Math. vol. 38, Part 1.

[15] A. Connes. Non-commutative differential geometry, I: the Chern character. Publ. Math. Inst. Hautes Etudes Sci., 62:41-93, 1986.

[16] A. Connes. Géométrie Non-commutative. InterEditions, Paris, 1990.

[17] A. Connes, M. Gromov, and H. Moscovici. Conjecture de Novikov et fibrés presque plats. C. R. Acad. Sci., Paris, 310:273-277, 1990.

[18] A. Connes, M. Gromov, and H. Moscovici. Hyper-linear cohomology and infinite groups. preprint, 1991.

[19] A. Connes and N. Higson. Déformations, morphismes asymptotiques et K-théorie bivariante. C. R. Acad. Sci., Paris, 311:101-106, 1990.

[20] A. Connes and N. Higson. Almost homomorphisms and K-theory. preprint, April, 1990.

[21] H. L. Cycon, R. G. Froese, W. Kirsch, and B. Simon. Schrödinger Operators, with Applications to Quantum Mechanics and Global Geometry. Springer-Verlag, New York and Berlin, 1987. Texts and Monographs in Physics.

[22] J.-P. Demailley. Champs magnétiques et inégalités de Morse pour la $d^{\prime \prime}$ cohomologie. Annales Inst. Fourier, Grenoble, 35:189-229, 1985.

[23] R.G. Douglas, S. Hurder, and J. Kaminker. Eta invariants and von Neumann algebras. Bulletin Amer. Math. Soc., 21:83-87, 1989.

[24] R.G. Douglas, S. Hurder, and J. Kaminker. Cyclic cocycles, renormalization and eta invariants. Invent. Math., 103:101-179, 1991.

[25] R.G. Douglas, S. Hurder, and J. Kaminker. The longitudinal cocycle and the index of Toeplitz operators. Jour. Func. Anal., 101:120-144, 1991.

[26] J. L. Dupont. Simplicial de Rham cohomology and characteristic classes of flat bundles. Topology, 15:233-245, 1976.

[27] M. Gromov. Groups of polynomial growth and expanding maps. Publ. Math. Inst. Hautes Etudes Sci., 53:53-78, 1981. 
[28] M. Gromov. Volume and bounded cohomology. Publ. Math. Inst. Hautes Etudes Sci., 56:5-100, 1982.

[29] M. Gromov and H. B. Lawson, Jr. Positive scalar curvature and the Dirac operator on complete riemannian manifolds. Publ. Math. Inst. Hautes Etudes Sci., 58:83-196, 1983

[30] M. Gromov and M. A. Shubin. Von Neumann spectra near zero. Geom. Anal. and Func. Anal., 1:375-404, 1991.

[31] A. Haefliger. Groupöides d'holonomie et classifiants. In Structures Transverses des Feuilletages. TOULOUSE 1982, pages 70-97, 1984. Astérisque No. 116.

[32] N. Higson. K-homology and operators on non-compact manifolds. preprint, 1987.

[33] M. Hilsum and G. Skandalis. Invariance par homotopie de la signature à coefficients dans un fibré presque plat. Jour. reine angew. Math., 423:73-99, 1992.

[34] S. Hurder. Eta invariants and the odd index theorem for coverings. In J. Kaminker, editor, Geometric and Topological Invariants of Elliptic Operators, pages 47-82, Providence, 1990. Amer. Math. Soc. Contemp. Math. vol. 105.

[35] S. Hurder. Secondary invariants and spectral theory of operators. Talk given at conference Applications of Algebraic Topology to Geometry and Analysis, M.S.R.I. Berkeley, January 1990.

[36] S. Hurder. Exotic cohomology for foliations. Talk given at conference K-homology and geometry. University of Colorado, Boulder, August 1991.

[37] S. Hurder. Analysis and Geometry of Foliations. 1992. Based on Ulam Lectures at the University of Colorado, 1988-89.

[38] S. Hurder. Exotic index theory for foliations. preprint, 1992.

[39] S. Hurder. Spectral theory of foliation geometric operators. in preparation, 1992.

[40] S. Hurder and A. Katok. Ergodic theory and Weil measures for foliations. Annals of Math., 126:221-275, 1987.

[41] H. B. Lawson, Jr. and M.-L. Michelsohn. Spin Geometry, volume 38 of Princeton Mathematical Series. Princeton University Press, Princeton, 1989.

[42] J. Lott. Heat kernels on covering spaces and topological invariants. Jour. Differential Geom., 1991.

[43] C. C. Moore and C. Schochet. Analysis on Foliated Spaces. Springer-Verlag, New York and Berlin, 1988. Math. Sci. Res. Inst. Publ. vol. 9.

[44] S. P. Novikov and M. A. Shubin. Morse theory and von Neumann invariants on non-simply connected manifolds. Uspekhi Matem. Nauk, 41:222-223, 1986. (in Russian).

[45] S. P. Novikov and M. A. Shubin. Morse theory and von Neumann $\mathrm{II}_{1}$-factors. Doklady Akad. Nauk SSSR, 289:289-292, 1986.

[46] J. Roe. Elliptic Operators, Topology and Asymptotic Methods. Res. Notes Math. Ser. 179. Pitman, 1988.

[47] J. Roe. Partitioning non-compact manifolds and the dual Toeplitz problem. In D. Evans and M. Takesaki, editors, Operator Algebras and Applications, pages 187-228. Cambridge University Press, 1989.

[48] J. Roe. Exotic cohomology and index theory. Bulletin Amer. Math. Soc., 23:447453, 1990. 
[49] J. Roe. Coarse cohomology and index theory on complete Riemannian manifolds. Memoires Amer. Math. Soc., 1992. preprint date-June, 1990.

[50] Y.-T. Siu. Asymptotic Morse inequalities for sheaf cohomology. In Séminaire BOURBAKI, pages 283-297, Paris, 1987. Société Mathématique de France. Astérisque vol. 145-46.

[51] W. Thurston. A norm for the homology of 3-manifolds, pages 99-130. Number 339 in Memoirs of the AMS. Amer. Math. Soc., 1986.

[52] C. Vafa and E. Witten. Eigenvalue inequalities for fermions in gauge theories. Comm. Math. Phys., 95:257-276, 1984.

[53] S.-T. Yau. Isoperimetric constants and the first eigenvalue of a compact Riemannian manifold. Ann. Sci. É. N. S., 8:487-507, 1975.

Department of Mathematics (M/C 249)

University of Illinois at ChicAGo

P. O. Box 4348

CHICAGO, IL 60680

E-mail address: hurder@math.uic.edu 